Supporting Information for

\title{
Ultra-High Proton Conduction via Extended Hydrogen-Bonding Network in a Preyssler-type Polyoxometalate-Based Framework Functionalized with Lanthanide Ion
}

Tsukasa Iwano, ${ }^{\dagger}$ Kota Shitamatsu, ${ }^{\ddagger}$ Naoki Ogiwara,${ }^{\dagger}$ Masanari Okuno, ${ }^{\dagger}$ Yuji Kikukawa, ${ }^{\S}$

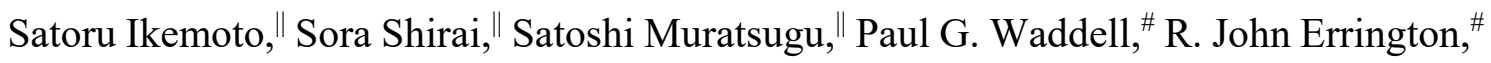
Masahiro Sadakane, ${ }^{*, *}$ and Sayaka Uchida ${ }^{*, \dagger}$

${ }^{\dagger}$ Department of Basic Science, School of Arts and Sciences, The University of Tokyo, 3-8-1 Komaba, Meguro-ku, Tokyo 153-8902, Japan. E-mail: csayaka@g.ecc.u-tokyo.ac.jp

\$ Department of Applied Chemistry, Graduate School of Advanced Science and Engineering, Hiroshima University, 1-4-1 Kagamiyama, Higashi-Hiroshima, Hiroshima 739-8527, Japan. E-mail: sadakane09@hiroshima-u.ac.jp

${ }^{\S}$ Department of Chemistry, Graduate School of Natural Science and Technology, Kanazawa University, Kakuma-machi, Kanazawa, Ishikawa 920-1192, Japan.

" Graduate School of Science, Nagoya University, Furo-cho, Chikusa-ku, Nagoya, Aichi 464-8602, Japan.

\# Department of Chemistry, School of Natural \& Environmental Sciences, Newcastle University, Newcastle upon Tyne, NE1 7RU, United Kingdom. 


\section{Table of Contents}

\begin{tabular}{|c|c|}
\hline Title & Pages \\
\hline Additional experimental details & S4 \\
\hline Table S1. Crystallographic data of I and II. & S5 \\
\hline Table S2. Selected bond distances $(\AA)$ of $\mathbf{I}$. & S6-S7 \\
\hline Table S3. Selected angles $\left(^{\circ}\right)$ and atomic numbering scheme of $\mathbf{I}$. & S8-S14 \\
\hline Table S4. Cell parameters of II calculated by Pawley fitting of PXRD. & S15 \\
\hline Table S5. Comparison of representative proton conductors. & S16 \\
\hline $\begin{array}{l}\text { Figure S1. PXRD patterns of III and crystalline composites composed of } \\
{\left[\mathrm{P}_{5} \mathrm{~W}_{30} \mathrm{O}_{110} \mathrm{Na}\left(\mathrm{H}_{2} \mathrm{O}\right)\right]^{14-} \text { and PAAs. }}\end{array}$ & S17 \\
\hline $\begin{array}{l}\text { Figure S2. }{ }^{31} \mathrm{P}-\mathrm{NMR} \text { spectra of (a) } \mathrm{K}_{12}\left[\mathrm{P}_{5} \mathrm{~W}_{30} \mathrm{O}_{110} \mathrm{Eu}\left(\mathrm{H}_{2} \mathrm{O}\right)\right] \cdot 24 \mathrm{H}_{2} \mathrm{O} \text {, (b) the solid } \\
\text { obtained after heating (a), and (c) } \mathbf{I} \text { in } \mathrm{D}_{2} \mathrm{O} \text {. }\end{array}$ & S17 \\
\hline $\begin{array}{l}\text { Figure S3. IR spectra of (a) } \mathrm{K}_{12}\left[\mathrm{P}_{5} \mathrm{~W}_{30} \mathrm{O}_{110} \mathrm{Eu}\left(\mathrm{H}_{2} \mathrm{O}\right)\right] \cdot 24 \mathrm{H}_{2} \mathrm{O} \text {, (b) the solid after } \\
\text { heating } \mathrm{K}_{12}\left[\mathrm{P}_{5} \mathrm{~W}_{30} \mathrm{O}_{110} \mathrm{Eu}\left(\mathrm{H}_{2} \mathrm{O}\right)\right] \cdot 24 \mathrm{H}_{2} \mathrm{O} \text {, (c) } \mathrm{K}_{14}\left[\mathrm{P}_{5} \mathrm{~W}_{30} \mathrm{O}_{110} \mathrm{~K}\right] \cdot 24 \mathrm{H}_{2} \mathrm{O} \text {, (d) I, and } \\
\text { (e) II. }\end{array}$ & S18 \\
\hline $\begin{array}{l}\text { Figure S4. }{ }^{31} \mathrm{P} \text { NMR spectra of (a) } \mathbf{I} \text {, (b) } \mathrm{H}_{14}\left[\mathrm{P}_{5} \mathrm{~W}_{30} \mathrm{O}_{110} \mathrm{~K}\right] \text {, and (c) after } \\
\text { neutralization of (b) with } \mathrm{K}_{2} \mathrm{CO}_{3} \text { in } \mathrm{D}_{2} \mathrm{O} \text {. }\end{array}$ & S19 \\
\hline $\begin{array}{l}\text { Figure S5. Left: }{ }^{31} \mathrm{P} \text { NMR spectra of (a) } \mathrm{K}_{14}\left[\mathrm{P}_{5} \mathrm{~W}_{30} \mathrm{O}_{110} \mathrm{~K}\right] \text { in } \mathrm{D}_{2} \mathrm{O} \text { and after } \\
\text { adding (b) } 0.1 \text {, (c) } 0.2 \text {, (d) } 0.3 \text {, (e) } 0.4 \text {, (f) } 0.5 \text {, and (g) } 1.0 \text { equivalent of } \mathrm{Eu}\left(\mathrm{NO}_{3}\right)_{3} \\
\text { at } 293 \mathrm{~K} \text {. Right: plot of } 1 / \Delta \text { against } 1 /[L] \text { (solid circles) and the regression line } \\
\text { (solid line) to estimate the binding constant } K \text { at } 293 \mathrm{~K} \text {. }\end{array}$ & S20 \\
\hline Figure S6. TG of (a) I, (b) II, and (c) III. & $\mathbf{S 2 1}$ \\
\hline $\begin{array}{l}\text { Figure S7. PXRD patterns of (a) I (calc), (b) I, (c) I (under saturated water vapor } \\
\text { condition), (d) } \mathrm{K}_{14}\left[\mathrm{P}_{5} \mathrm{~W}_{30} \mathrm{O}_{110} \mathrm{~K}\right] \text {, (e) II (calc), (f) II, and (g) II (under saturated } \\
\text { water vapor condition). }\end{array}$ & $\mathbf{S 2 1}$ \\
\hline $\begin{array}{l}\text { Figure S8. }{ }^{1} \mathrm{H} \text { MAS NMR spectra (MAS }=10 \mathrm{kHz} \text { ) of I (black) and } \mathbf{I I} \text { (red). (b) } \\
{ }^{13} \mathrm{C} \text { CP MAS NMR spectrum (MAS }=5 \mathrm{kHz} \text { ) of } \mathbf{I I} .\end{array}$ & S22 \\
\hline $\begin{array}{l}\text { Figure S9. Raman/luminescence emission spectra of } \mathbf{I} \text { (black) and II (red), } \\
\text { together with those of } \mathrm{Eu}_{2} \mathrm{O}_{3} \text { (blue) and } \mathrm{Eu}\left(\mathrm{NO}_{3}\right) \cdot 6 \mathrm{H}_{2} \mathrm{O} \text { (green) as references. }\end{array}$ & $\mathbf{S 2 3}$ \\
\hline
\end{tabular}




\begin{tabular}{|c|c|}
\hline $\begin{array}{l}\text { Figure S10. (a) } k^{3} \text {-Weighed } \mathrm{Eu} \mathrm{L}_{\mathrm{III}} \text {-edge EXAFS oscillations and (b) their } \\
\text { Fourier transforms }\left(k=30-120 \mathrm{~nm}^{-1}, R=0.11-0.29 \mathrm{~nm}\right) \text { for } \mathbf{I} \text { and } \mathbf{I I} \text {. }\end{array}$ & S23 \\
\hline $\begin{array}{l}\text { Figure S11. Nyquist plots (solid circles) of the impedance spectra along with the } \\
\text { fitting with an electrical equivalent circuit (see experimental section, solid lines) } \\
\text { of a pelleted sample of II at } 368 \mathrm{~K} \text { and RH of (a) } 90 \% \text {, (b) } 75 \% \text {, (c) } 50 \% \text {, and } \\
\text { (d) } 30 \% \text {. }\end{array}$ & S24 \\
\hline $\begin{array}{l}\text { Figure S12. PXRD patterns of II (a) before and (b) after heating at } 368 \mathrm{~K} \text { and } \\
90 \% \mathrm{RH} .\end{array}$ & S25 \\
\hline Figure S13. Water sorption-desorption isotherms of III. & S25 \\
\hline $\begin{array}{l}\text { Figure S14. Changes in the water sorption enthalpy of I, II, and III with } \mathrm{H}_{2} \mathrm{O} \\
\text { uptake. }\end{array}$ & S26 \\
\hline $\begin{array}{l}\text { Figure S15. In situ PXRD patterns of II measured by the parallel beam method } \\
\text { at } 303 \mathrm{~K} \text {. (a)(b) As synthesized, (c) dry } \mathrm{N}_{2} \text {, (d) RH } 30 \% \text { (e) RH } 50 \% \text {, (f) RH } \\
75 \% \text {, and (g) RH ca. } 100 \% \text {. }\end{array}$ & S26 \\
\hline $\begin{array}{l}\text { Figure S16. Experimental and calculated PXRD patterns of } \mathbf{I I} \text { under } \mathrm{N}_{2} \text { by the } \\
\text { Pawley method. The bottom line shows the difference profile. }\end{array}$ & S27 \\
\hline $\begin{array}{l}\text { Figure S17. Experimental and calculated PXRD patterns of II at RH of } 30 \% \text { by } \\
\text { the Pawley method. The bottom line shows the difference profile. }\end{array}$ & S27 \\
\hline $\begin{array}{l}\text { Figure S18. Experimental and calculated PXRD patterns of II at RH of } 50 \% \text { by } \\
\text { the Pawley method. The bottom line shows the difference profile. }\end{array}$ & S28 \\
\hline $\begin{array}{l}\text { Figure S19. Experimental and calculated PXRD patterns of II at RH of } 75 \% \text { by } \\
\text { the Pawley method. The bottom line shows the difference profile. }\end{array}$ & S28 \\
\hline $\begin{array}{l}\text { Figure S20. Experimental and calculated PXRD patterns of II at RH of ca. } 100 \% \\
\text { by the Pawley method. The bottom line shows the difference profile. }\end{array}$ & S29 \\
\hline $\begin{array}{l}\text { Figure S21. Background-subtracted IR spectra }(293 \mathrm{~K}) \text { of II under a water vapor } \\
\text { pressure of (a) } 0.5 \mathrm{kPa} \text {, (b) } 1.0 \mathrm{kPa} \text {, (c) } 1.5 \mathrm{kPa} \text {, and (d) } 2.0 \mathrm{kPa} \text { in the } \mathrm{OH} \\
\text { stretching region. }\end{array}$ & S30 \\
\hline References & S31-S32 \\
\hline
\end{tabular}




\section{Additional experimental details}

Materials. A 40\% aqueous solution of poly(allylamine hydrochloride) $(m=3000)$ was provided from Nittobo Medical Co., Ltd. All other chemicals were reagent grade and used as supplied. Homemade deionized water (Millipore, Elix) was used.

Measurements. Infrared (IR) spectra were recorded on a NICOLET 6700 Fourier transforms (FT)-IR spectrometer (Thermo Fisher Scientific) or a JASCO FT-IR 4100 spectrometer (JASCO) using $\mathrm{KBr}$ pellets. ${ }^{31} \mathrm{P}$ nuclear magnetic resonance (NMR) spectra were recorded in $\mathrm{D}_{2} \mathrm{O}$ on a Varian (Agilent) $500 \mathrm{MHz}$ spectrometer $\left({ }^{31} \mathrm{P}\right.$ resonance frequency: $202.333 \mathrm{MHz}$ ). The spectra were referenced to external $85 \% \mathrm{H}_{3} \mathrm{PO}_{4}(0 \mathrm{ppm})$. PXRD patterns were measured with a new advance D8 X-ray diffractometer (Bruker) using $\mathrm{Cu} \mathrm{K} \alpha$ radiation $(\lambda=1.54056 \AA, 40 \mathrm{kV}, 40 \mathrm{~mA})$ at $2 \theta=3-20^{\circ}$ and $1.8^{\circ} \mathrm{min}^{-1}$.

Elemental analysis of II. Combustion analysis (Elementar, vario MICRO cube) was used for the quantitative analysis of $\mathrm{C}, \mathrm{H}$, and $\mathrm{N}$. Inductively coupled plasma optical emission spectrometry (ICP-OES) (Agilent Technologies, ICP-OES720) was used for the quantitative analysis of Eu, P, and W. AAS (Hitachi, ZA3000) was performed for the quantitative analysis of K. Prior to the ICP-OES and AAS analysis, ca. $10 \mathrm{mg}$ of the solid (accurately weighed) was dispersed in $10 \mathrm{~mL}$ of $\mathrm{H}_{2} \mathrm{O}, 10 \mathrm{~mL}$ of conc. $\mathrm{HNO}_{3}$ was added, and the solution was heated to dissolve the solid completely. Then, the solution was diluted up to exactly $100 \mathrm{~mL}$.

Single-crystal X-ray diffraction (SXRD) measurements. Data for the crystal structure of I were collected at $150 \mathrm{~K}$ on a Rigaku Oxford Diffraction Xcalibur Gemini Ultra diffractometer equipped with an Oxford Cryosystems open-flow $\mathrm{N}_{2}$ cooling device using $\mathrm{Cu} \mathrm{K} \alpha$ radiation $(\lambda=1.54184$ $\AA$ ). The intensities were corrected for absorption using a multifaceted crystal model created by indexing the faces of the crystal for which data were collected. Data for the crystal structure of II were collected at $153 \mathrm{~K}$ with a CCD 2-D detector using a Rigaku Saturn diffractometer with graphite monochromated Mo K $\alpha$ radiation $(\lambda=0.71073 \AA)$.

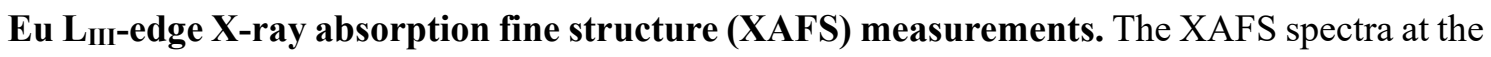
Eu L $\mathrm{L}_{\text {III }}$ edge were measured in transmission mode at the BL11S2 station at Aichi Synchrotron Radiation Center (Aichi, Japan). The energy and current of electrons in the storage ring were 1.2 $\mathrm{GeV}$ and $300 \mathrm{~mA}$, respectively. X-rays from the storage ring were monochromatized using a $\mathrm{Si}(111)$ double-crystal monochromator, and ionization chambers filled with pure $\mathrm{He} / \mathrm{N}_{2}(20 / 80)$ and $\mathrm{N}_{2} / \mathrm{Ar}(90 / 10)$ gases and a seven-channel silicon drift detector were used to monitor the incident and transmitted X-rays, respectively.

Alternating current (AC) impedance measurements. About $0.3 \mathrm{~g}$ of each compound was compressed at $190 \mathrm{kgf} \mathrm{cm}^{-2}$ into pellets of $10 \mathrm{~mm}$ in diameter and ca. $1.0 \mathrm{~mm}$ in thickness. The pellets were cut into fourths, and one-fourth of the pellet was used for the AC impedance measurement. AC impedance measurements were conducted in a temperature and humidity chamber with a BioLogic VMP3 multichannel potentiostat/galvanostat (Science Instruments) over a frequency range of $2 \mathrm{~Hz}$ to $7 \mathrm{MHz}$ and an $\mathrm{AC}$ amplitude of $100 \mathrm{mV}$. Gold electrodes with copper wire were attached on both faces of the pellets. 
Table S1. Crystallographic data of I and II.

\begin{tabular}{|c|c|c|}
\hline & $\mathbf{I}$ & II \\
\hline Crystal system & orthorhombic & triclinic \\
\hline Space group & Pnma & $P-1$ \\
\hline \multirow{6}{*}{ Unit cell } & $a=22.1603(2)$ & $a=21.283(4)$ \\
\hline & $b=21.1759(2)$ & $b=22.187(4)$ \\
\hline & \multirow[t]{4}{*}{$c=30.0914(3)$} & $c=30.168$ \\
\hline & & $\alpha=90.059(4)$ \\
\hline & & $\beta=90.010(4)$ \\
\hline & & $\gamma=90.109(4)$ \\
\hline Volume & $14120.8(2)$ & $14245(4)$ \\
\hline$Z$ & 4 & 4 \\
\hline$D_{\text {calc }}\left(\mathrm{g} \mathrm{cm}^{-3}\right)$ & 4.024 & 3.846 \\
\hline$F(000)$ & 14873 & 14272 \\
\hline$h, k, l$ range & $\begin{array}{l}-26 / 26,-25 / 20 \\
-35 / 27\end{array}$ & $\begin{array}{l}-33 / 29,-32 / 31, \\
-43 / 43\end{array}$ \\
\hline$\mu(\mathrm{Mo} \mathrm{K} \alpha)\left(\mathrm{mm}^{-1}\right)$ & 51.429 & 24.911 \\
\hline$R_{1}(I>2 \sigma(I))$ & 0.0829 & 0.1969 \\
\hline$w R_{2}$ (all data) & 0.1963 & 0.4520 \\
\hline GOF on $F^{2}$ & 1.150 & 1.108 \\
\hline
\end{tabular}


Table S2. Selected bond distances $(\AA)$ of $\mathbf{I}$.

\begin{tabular}{|c|c|c|c|c|c|}
\hline \multicolumn{6}{|c|}{ Compound I } \\
\hline $\mathrm{W} 1-\mathrm{O} 1$ & $1.73(3)$ & W6-O27 & $1.97(2)$ & W11-O38 & $2.268(17)$ \\
\hline W1-O17 & $1.954(18)$ & W6-O28 & $1.903(19)$ & W11-O39 & $1.927(18)$ \\
\hline W1-O18 & $1.882(18)$ & W6-O38 & $2.259(18)$ & W11-O49 & $1.964(18)$ \\
\hline W1-O31 & $2.17(2)$ & W6-O39 & $1.895(18)$ & W11-O50 & $1.948(18)$ \\
\hline W2-K2 & $4.047(11)$ & W7-K4 & $3.695(12)$ & W11-O55 & $1.891(19)$ \\
\hline $\mathrm{W} 2-\mathrm{O} 2$ & $1.73(2)$ & W7-O7 & $1.72(2)$ & W12-K5 & $3.908(10)$ \\
\hline W2-O18 & $1.890(17)$ & W7-O24 & $1.904(18)$ & W12-O12 & $1.70(2)$ \\
\hline W2-O19 & $1.904(18)$ & W7-O28 & $1.955(19)$ & $\mathrm{W} 12-\mathrm{O} 40$ & $2.256(16)$ \\
\hline $\mathrm{W} 2-\mathrm{O} 22$ & $1.912(18)$ & W7-O29 & $1.938(19)$ & $\mathrm{W} 12-\mathrm{O} 42$ & $1.928(18)$ \\
\hline $\mathrm{W} 2-\mathrm{O} 23$ & $1.928(17)$ & W7-O40 & $2.272(16)$ & $\mathrm{W} 12-\mathrm{O} 50$ & $1.920(17)$ \\
\hline W2-O32 & $2.184(17)$ & W7-O42 & $1.934(18)$ & W12-O51 & $1.968(18)$ \\
\hline W3-K2 & $3.930(9)$ & W8-K4 & $3.950(11)$ & W12-O56 & $1.867(17)$ \\
\hline W3-K3 & $4.073(6)$ & W8-O8 & $1.70(2)$ & W13-O13 & $1.714(19)$ \\
\hline W3-O3 & $1.71(2)$ & W8-O21 & $1.861(17)$ & W13-O41 & $2.263(16)$ \\
\hline W3-O19 & $1.879(18)$ & W8-O29 & $1.973(19)$ & W13-O43 & $1.908(19)$ \\
\hline W3-O20 & $1.890(5)$ & W8-O30 & $1.929(4)$ & W13-O51 & $1.951(19)$ \\
\hline W3-O21 & $1.951(18)$ & W8-O41 & $2.241(17)$ & W13-O52 & $1.941(4)$ \\
\hline W3-O24 & $1.908(18)$ & W8-O43 & $1.918(19)$ & W13-O59 & $1.854(17)$ \\
\hline W3-O33 & $2.151(17)$ & W9-O9 & $1.67(2)$ & W14-O14 & $1.74(3)$ \\
\hline W4-O4 & $1.725(19)$ & W9-O34 & $1.92(2)$ & W14-O44 & $2.20(2)$ \\
\hline W4-O17 & $1.846(19)$ & W9-O35 & $2.270(17)$ & W14-O53 & $1.917(18)$ \\
\hline W4-O25 & $1.962(7)$ & W9-O47 & $1.954(7)$ & W14-O57 & $1.928(18)$ \\
\hline W4-O26 & $1.942(19)$ & W9-O48 & $1.922(19)$ & W15-K6 & $3.700(12)$ \\
\hline W4-O34 & $1.94(2)$ & W9-O53 & $1.892(19)$ & W15-O15 & $1.71(2)$ \\
\hline W4-O35 & $2.253(17)$ & W10-O10 & $1.73(2)$ & $\mathrm{W} 15-\mathrm{O} 45$ & $2.157(17)$ \\
\hline W5-O5 & $1.70(2)$ & W10-O36 & $1.914(18)$ & W15-O54 & $1.923(19)$ \\
\hline W5-O22 & $1.871(18)$ & W10-O37 & $2.257(18)$ & W15-O55 & $1.922(18)$ \\
\hline W5-O26 & $1.94(2)$ & W10-O48 & $1.95(2)$ & W15-O57 & $1.847(18)$ \\
\hline W5-O27 & $1.93(2)$ & W10-O49 & $1.955(18)$ & W15-O58 & $1.876(17)$ \\
\hline W5-O36 & $1.893(18)$ & W10-O54 & $1.880(19)$ & W16-O16 & $1.745(17)$ \\
\hline W5-O37 & $2.269(18)$ & W11-K5 & $3.922(10)$ & W16-O46 & $2.172(17)$ \\
\hline W6-O6 & $1.72(2)$ & W11-K6 & $3.796(13)$ & W16-O56 & $1.936(17)$ \\
\hline W6-O23 & $1.873(18)$ & W11-O11 & $1.70(2)$ & W16-O58 & $1.901(17)$ \\
\hline
\end{tabular}




\begin{tabular}{llllll}
\hline W16-O59 & $1.942(17)$ & K2-O72B & $2.91(4)$ & K5-O75 & $3.32(3)$ \\
W16-O60 & $1.892(5)$ & K2-O72A & $2.49(4)$ & K5-O77 & $3.349(12)$ \\
Eu1-O1 & $2.52(3)$ & K3-O3 & $2.37(2)$ & K5-O76A & $3.08(5)$ \\
Eu1-O61 & $2.39(5)$ & K3-O70 & $2.84(4)$ & K5-O76B & $2.63(7)$ \\
Eu1-O62 & $2.38(3)$ & K3-O71A & $2.601(11)$ & K6-O11 & $3.07(2)$ \\
Eu1-O63 & $2.49(3)$ & K3-O68A & $3.42(8)$ & K6-O15 & $2.69(2)$ \\
Eu1-O64 & $2.38(4)$ & K3-O69A & $2.37(5)$ & K6-O55 & $2.97(2)$ \\
Eu1-O65B & $2.45(6)$ & K3-O71B & $3.11(7)$ & K6-O77 & $2.669(14)$ \\
Eu1-O65A & $2.44(6)$ & K3-O72B & $2.49(4)$ & K6-O78 & $2.66(4)$ \\
K1-P2 & $3.535(6)$ & K3-O68B & $2.32(6)$ & K6-O79 & $2.82(4)$ \\
K1-O31 & $2.97(2)$ & K3-O69B & $2.87(6)$ & P1-O33 & $1.534(17)$ \\
K1-O32 & $2.927(17)$ & K3-O72A & $2.91(4)$ & P1-O40 & $1.524(18)$ \\
K1-O33 & $2.933(18)$ & K4-O7 & $2.73(2)$ & P1-O41 & $1.574(18)$ \\
K1-O44 & $2.92(3)$ & K4-O8 & $3.10(2)$ & P1-O46 & $1.506(17)$ \\
K1-O45 & $2.955(17)$ & K4-O29 & $2.94(2)$ & P2-O32 & $1.526(17)$ \\
K1-O46 & $2.950(18)$ & K4-O73 & $2.73(4)$ & P2-O37 & $1.529(18)$ \\
K2-K3 & K2-K3 & K4-O69B & $2.89(6)$ & P2-O38 & $1.517(18)$ \\
K2-O2 & K2-O2 & K5-O11 & $2.86(2)$ & P2-O45 & $1.509(17)$ \\
K2-O3 & K2-O3 & K5-O12 & $2.83(2)$ & P3-O31 & $1.53(3)$ \\
K2-O66 & K2-O66 & K5-O50 & $3.30(2)$ & P3-O35 & $1.527(17)$ \\
K2-O67 & K2-O67 & K5-O74 & $2.81(2)$ & P3-O44 & $1.49(3)$ \\
\hline
\end{tabular}


Table S3. Selected angles $\left(^{\circ}\right)$ and atomic numbering scheme of $\mathbf{I}$.

\begin{tabular}{|c|c|c|c|c|c|}
\hline \multicolumn{6}{|l|}{ Compound I } \\
\hline O1-W1-O17 & $94.9(9)$ & O3-W3-O24 & $95.7(8)$ & O26-W4-O35 & $86.1(7)$ \\
\hline O1-W1-O18 & $97.6(8)$ & O3-W3-O33 & $177.4(8)$ & O34-W4-O25 & $89.5(11)$ \\
\hline $\mathrm{O} 1-\mathrm{W} 1-\mathrm{O} 31$ & $178.6(11)$ & O19-W3-K2 & $61.7(5)$ & O34-W4-O26 & $89.9(9)$ \\
\hline O17-W1-O31 & $84.1(7)$ & O19-W3-K3 & $101.5(5)$ & O34-W4-O35 & $73.5(7)$ \\
\hline O18-W1-O17 & $90.5(8)$ & O19-W3-O20 & $89.4(10)$ & O5-W5-O22 & 103.2(9) \\
\hline O18-W1-O31 & $83.4(7)$ & O19-W3-O21 & $168.2(7)$ & O5-W5-O26 & $96.7(8)$ \\
\hline $\mathrm{O} 2-\mathrm{W} 2-\mathrm{K} 2$ & $38.3(7)$ & O19-W3-O24 & $90.3(7)$ & O5-W5-O27 & $95.6(9)$ \\
\hline O2-W2-O18 & $97.9(8)$ & O19-W3-O33 & $84.2(7)$ & O5-W5-O36 & $99.6(8)$ \\
\hline O2-W2-O19 & $96.9(8)$ & $\mathrm{O} 20-\mathrm{W} 3-\mathrm{K} 2$ & $99.2(7)$ & O5-W5-O37 & $171.6(8)$ \\
\hline $\mathrm{O} 2-\mathrm{W} 2-\mathrm{O} 22$ & $96.6(8)$ & $\mathrm{O} 20-\mathrm{W} 3-\mathrm{K} 3$ & $100.6(8)$ & $\mathrm{O} 22-\mathrm{W} 5-\mathrm{O} 26$ & $89.2(8)$ \\
\hline $\mathrm{O} 2-\mathrm{W} 2-\mathrm{O} 23$ & $95.5(8)$ & $\mathrm{O} 20-\mathrm{W} 3-\mathrm{O} 21$ & $90.5(9)$ & $\mathrm{O} 22-\mathrm{W} 5-\mathrm{O} 27$ & $84.5(8)$ \\
\hline $\mathrm{O} 2-\mathrm{W} 2-\mathrm{O} 32$ & $177.5(8)$ & $\mathrm{O} 20-\mathrm{W} 3-\mathrm{O} 24$ & $166.4(9)$ & $\mathrm{O} 22-\mathrm{W} 5-\mathrm{O} 36$ & 157.1(7) \\
\hline O18-W2-K2 & $99.1(6)$ & $\mathrm{O} 20-\mathrm{W} 3-\mathrm{O} 33$ & $82.6(9)$ & O22-W5-O37 & $84.7(7)$ \\
\hline O18-W2-O19 & $91.3(7)$ & $\mathrm{O} 21-\mathrm{W} 3-\mathrm{K} 2$ & $129.8(5)$ & O26-W5-O37 & $86.0(7)$ \\
\hline $\mathrm{O} 18-\mathrm{W} 2-\mathrm{O} 22$ & $90.8(7)$ & $\mathrm{O} 21-\mathrm{W} 3-\mathrm{K} 3$ & $90.1(5)$ & $\mathrm{O} 27-\mathrm{W} 5-\mathrm{O} 26$ & $167.2(8)$ \\
\hline $\mathrm{O} 18-\mathrm{W} 2-\mathrm{O} 23$ & $166.5(7)$ & $\mathrm{O} 21-\mathrm{W} 3-\mathrm{O} 33$ & $84.1(7)$ & O27-W5-O37 & $82.3(7)$ \\
\hline $\mathrm{O} 18-\mathrm{W} 2-\mathrm{O} 32$ & $84.6(7)$ & $\mathrm{O} 24-\mathrm{W} 3-\mathrm{K} 2$ & $92.7(5)$ & O36-W5-O26 & $90.1(8)$ \\
\hline O19-W2-K2 & $58.6(5)$ & O24-W3-K3 & $92.8(5)$ & O36-W5-O27 & $91.4(8)$ \\
\hline O19-W2-O22 & $165.9(7)$ & O24-W3-O21 & $86.9(7)$ & O36-W5-O37 & $72.4(7)$ \\
\hline O19-W2-O23 & $89.1(7)$ & $\mathrm{O} 24-\mathrm{W} 3-\mathrm{O} 33$ & $83.9(7)$ & O6-W6-O23 & $102.4(8)$ \\
\hline O19-W2-O32 & $82.8(7)$ & O33-W3-K2 & $145.7(5)$ & O6-W6-O27 & $96.4(9)$ \\
\hline $\mathrm{O} 22-\mathrm{W} 2-\mathrm{K} 2$ & $134.7(5)$ & O33-W3-K3 & $173.5(5)$ & O6-W6-O28 & $95.7(8)$ \\
\hline $\mathrm{O} 22-\mathrm{W} 2-\mathrm{O} 23$ & $85.7(7)$ & O4-W4-O17 & $102.4(9)$ & O6-W6-O38 & $172.7(8)$ \\
\hline $\mathrm{O} 22-\mathrm{W} 2-\mathrm{O} 32$ & $83.5(7)$ & O4-W4-O25 & $97.4(10)$ & O6-W6-O39 & $99.2(9)$ \\
\hline $\mathrm{O} 23-\mathrm{W} 2-\mathrm{K} 2$ & $92.7(5)$ & O4-W4-O26 & $95.8(8)$ & O23-W6-O27 & $85.3(8)$ \\
\hline $\mathrm{O} 23-\mathrm{W} 2-\mathrm{O} 32$ & $82.1(7)$ & O4-W4-O34 & $97.9(9)$ & O23-W6-O28 & $89.3(7)$ \\
\hline $\mathrm{O} 32-\mathrm{W} 2-\mathrm{K} 2$ & $141.2(5)$ & O4-W4-O35 & $171.2(8)$ & O23-W6-O38 & $84.6(7)$ \\
\hline K2-W3-K3 & $39.77(18)$ & O17-W4-O25 & $86.1(10)$ & O23-W6-O39 & $158.4(7)$ \\
\hline O3-W3-K2 & $36.8(7)$ & O17-W4-O26 & $89.9(8)$ & O27-W6-O38 & $82.0(7)$ \\
\hline O3-W3-K3 & $4.2(7)$ & O17-W4-O34 & $159.6(8)$ & O28-W6-O27 & $167.6(8)$ \\
\hline O3-W3-O19 & $98.4(8)$ & O17-W4-O35 & $86.2(7)$ & O28-W6-O38 & $86.3(7)$ \\
\hline O3-W3-O20 & $97.8(10)$ & $\mathrm{O} 25-\mathrm{W} 4-\mathrm{O} 35$ & $81.0(9)$ & O39-W6-O27 & $91.6(8)$ \\
\hline O3-W3-O21 & $93.3(8)$ & $\mathrm{O} 26-\mathrm{W} 4-\mathrm{O} 25$ & $166.7(9)$ & O39-W6-O28 & $89.4(7)$ \\
\hline
\end{tabular}




\begin{tabular}{|c|c|c|c|c|c|}
\hline O39-W6-O38 & $73.8(7)$ & O30-W8-O29 & $166.6(9)$ & O54-W10-O49 & $87.6(8)$ \\
\hline O7-W7-K4 & $43.8(7)$ & O30-W8-O41 & $84.7(9)$ & K6-W11-K5 & $67.8(3)$ \\
\hline O7-W7-O24 & $103.1(8)$ & O41-W8-K4 & $128.0(5)$ & O11-W11-K5 & $40.8(7)$ \\
\hline O7-W7-O28 & $97.5(8)$ & O43-W8-K4 & $94.6(6)$ & O11-W11-K6 & $52.4(7)$ \\
\hline O7-W7-O29 & $96.1(8)$ & O43-W8-O29 & $89.1(8)$ & O11-W11-O38 & $172.4(8)$ \\
\hline O7-W7-O40 & $171.8(8)$ & O43-W8-O30 & $91.6(9)$ & O11-W11-O39 & $100.0(8)$ \\
\hline O7-W7-O42 & $98.0(8)$ & O43-W8-O41 & $73.1(7)$ & O11-W11-O49 & $95.6(8)$ \\
\hline O24-W7-K4 & $100.3(5)$ & O9-W9-O34 & $98.5(9)$ & O11-W11-O50 & $97.9(8)$ \\
\hline $\mathrm{O} 24-\mathrm{W} 7-\mathrm{O} 28$ & $88.4(7)$ & O9-W9-O35 & $171.6(8)$ & O11-W11-O55 & $101.5(9)$ \\
\hline O24-W7-O29 & $86.2(7)$ & O9-W9-O47 & $96.8(11)$ & O38-W11-K5 & $142.0(5)$ \\
\hline O24-W7-O40 & $84.8(6)$ & O9-W9-O48 & $97.0(9)$ & O38-W11-K6 & $133.7(5)$ \\
\hline O24-W7-O42 & $158.9(7)$ & O9-W9-O53 & $102.4(9)$ & O39-W11-K5 & $100.7(5)$ \\
\hline O28-W7-K4 & $141.3(5)$ & O34-W9-O35 & $73.4(7)$ & O39-W11-K6 & $149.5(5)$ \\
\hline $\mathrm{O} 28-\mathrm{W} 7-\mathrm{O} 40$ & $85.0(6)$ & O34-W9-O47 & $90.8(11)$ & O39-W11-O38 & $73.0(7)$ \\
\hline O29-W7-K4 & $52.4(6)$ & O34-W9-O48 & $89.9(9)$ & O39-W11-O49 & $89.9(7)$ \\
\hline O29-W7-O28 & $166.2(7)$ & O47-W9-O35 & $81.3(9)$ & O39-W11-O50 & $89.4(7)$ \\
\hline O29-W7-O40 & $81.9(7)$ & O48-W9-O35 & $85.4(7)$ & O49-W11-K5 & $136.2(5)$ \\
\hline O40-W7-K4 & $133.1(5)$ & O48-W9-O47 & $165.9(10)$ & O49-W11-K6 & $81.6(6)$ \\
\hline O42-W7-K4 & $94.0(5)$ & O53-W9-O34 & 159.1 & O49-W11-O38 & $81.8(7)$ \\
\hline $\mathrm{O} 42-\mathrm{W} 7-\mathrm{O} 28$ & $90.1(7)$ & O53-W9-O35 & $85.7(7)$ & O50-W11-K5 & $57.1(5)$ \\
\hline O42-W7-O29 & $90.4(7)$ & O53-W9-O47 & $86.3(10)$ & O50-W11-K6 & $105.5(6)$ \\
\hline $\mathrm{O} 42-\mathrm{W} 7-\mathrm{O} 40$ & $74.1(6)$ & O53-W9-O48 & $88.1(8)$ & O50-W11-O38 & $85.0(7)$ \\
\hline O8-W8-K4 & $48.7(7)$ & O10-W10-O36 & $97.9(8)$ & O50-W11-O49 & $166.4(7)$ \\
\hline $\mathrm{O} 8-\mathrm{W} 8-\mathrm{O} 21$ & 101.3(9) & O10-W10-O37 & $170.1(8)$ & O55-W11-K5 & $96.6(6)$ \\
\hline O8-W8-O29 & $94.6(9)$ & O10-W10-O48 & $96.9(9)$ & O55-W11-K6 & $50.5(6)$ \\
\hline $\mathrm{O} 8-\mathrm{W} 8-\mathrm{O} 30$ & $98.4(10)$ & O10-W10-O49 & $96.7(8)$ & O55-W11-O38 & $85.5(7)$ \\
\hline O8-W8-O41 & $172.8(8)$ & O10-W10-O54 & $105.0(8)$ & O55-W11-O39 & $158.5(8)$ \\
\hline O8-W8-O43 & $100.3(9)$ & O36-W10-O37 & $72.3(7)$ & O55-W11-O49 & $86.4(7)$ \\
\hline O21-W8-K4 & $97.8(6)$ & O36-W10-O48 & $88.6(8)$ & O55-W11-O50 & $89.4(7)$ \\
\hline O21-W8-O29 & $86.8(7)$ & O36-W10-O49 & $89.7(7)$ & O12-W12-K5 & $40.2(7)$ \\
\hline $\mathrm{O} 21-\mathrm{W} 8-\mathrm{O} 30$ & $87.6(9)$ & O48-W10-O37 & $84.2(7)$ & O12-W12-O40 & $172.4(8)$ \\
\hline O21-W8-O41 & $85.3(7)$ & O48-W10-O49 & $166.4(7)$ & O12-W12-O42 & $97.9(8)$ \\
\hline O21-W8-O43 & $158.3(8)$ & O49-W10-O37 & $82.4(7)$ & O12-W12-O50 & $97.5(8)$ \\
\hline O29-W8-K4 & $46.0(5)$ & O54-W10-O36 & $157.1(7)$ & O12-W12-O51 & $95.9(8)$ \\
\hline O29-W8-O41 & $82.7(7)$ & O54-W10-O37 & $84.8(7)$ & O12-W12-O56 & $102.1(8)$ \\
\hline O30-W8-K4 & $147.1(8)$ & O54-W10-O48 & $88.7(8)$ & O40-W12-K5 & $141.2(5)$ \\
\hline
\end{tabular}




\begin{tabular}{|c|c|c|c|c|c|}
\hline O42-W12-K5 & $99.4(6)$ & O15-W15-O45 & $176.4(8)$ & O61-Eu1-O63 & $135.4(13)$ \\
\hline O42-W12-O40 & $74.6(7)$ & O15-W15-O54 & $94.4(8)$ & O61-Eu1-O65B & $72(2)$ \\
\hline O42-W12-O51 & $90.5(7)$ & O15-W15-O55 & $93.9(9)$ & O61-Eu1-O65A & $67(2)$ \\
\hline O50-W12-K5 & $57.5(6)$ & O15-W15-O57 & $98.1(9)$ & O62-Eu1-O1 & $75.1(11)$ \\
\hline O50-W12-O40 & $84.0(7)$ & O15-W15-O58 & $97.6(8)$ & O62-Eu1-O61 & $90.3(12)$ \\
\hline O50-W12-O42 & $89.4(7)$ & O45-W15-K6 & $134.9(5)$ & O62-Eu1-O63 & $133.0(17)$ \\
\hline O50-W12-O51 & $166.5(7)$ & O54-W15-K6 & $81.2(6)$ & O62-Eu1-O64 & $67.5(10)$ \\
\hline O51-W12-K5 & $135.7(5)$ & O54-W15-O45 & $82.6(7)$ & O62-Eu1-O65B & $92(3)$ \\
\hline O51-W12-O40 & $83.0(6)$ & O55-W15-K6 & $53.1(6)$ & O62-Eu1-O65A & $61(2)$ \\
\hline O56-W12-K5 & $96.7(5)$ & O55-W15-O45 & $84.0(7)$ & O63-Eu1-O1 & $72.7(10)$ \\
\hline O56-W12-O40 & $85.4(7)$ & O55-W15-O54 & $85.9(7)$ & O64-Eu1-O1 & $72.6(13)$ \\
\hline O56-W12-O42 & $160.0(7)$ & O57-W15-K6 & $137.8(6)$ & O64-Eu1-O61 & $69.3(17)$ \\
\hline O56-W12-O50 & $89.3(7)$ & O57-W15-O45 & $83.9(7)$ & O64-Eu1-O63 & $130.4(13)$ \\
\hline O56-W12-O51 & $86.3(7)$ & O57-W15-O54 & $90.4(7)$ & O64-Eu1-O65B & $136(2)$ \\
\hline O13-W13-O41 & $172.7(8)$ & O57-W15-O55 & $167.7(8)$ & O64-Eu1-O65A & $109(2)$ \\
\hline O13-W13-O43 & $100.0(9)$ & O57-W15-O58 & $91.0(7)$ & $\mathrm{O} 65 \mathrm{~B}-\mathrm{Eu}$ & $142(2)$ \\
\hline O13-W13-O51 & $95.6(8)$ & O58-W15-K6 & $105.7(6)$ & O65B-Eu1-O63 & $92(3)$ \\
\hline O13-W13-O52 & $97.0(10)$ & O58-W15-O45 & $85.3(7)$ & O65A-Eu1-O1 & $129(2)$ \\
\hline O13-W13-O59 & $102.2(8)$ & O58-W15-O54 & $167.6(7)$ & O65A-Eu1-O63 & $120(2)$ \\
\hline O43-W13-O41 & $72.7(7)$ & O58-W15-O55 & $90.0(7)$ & $\mathrm{P} 21-\mathrm{K} 1-\mathrm{P} 2$ & $143.8(3)$ \\
\hline O43-W13-O51 & $89.0(7)$ & O16-W16-O46 & $177.2(7)$ & $\mathrm{O} 31-\mathrm{K} 1-\mathrm{P} 2$ & $73.65(17)$ \\
\hline O43-W13-O52 & $90.2(9)$ & O16-W16-O56 & $94.7(7)$ & $\mathrm{O} 32-\mathrm{K} 1-\mathrm{P} 2$ & 25.1(3) \\
\hline O51-W13-O41 & $83.8(7)$ & O16-W16-O58 & $97.6(8)$ & $\mathrm{O} 32-\mathrm{K} 1-\mathrm{O} 31$ & $64.3(4)$ \\
\hline O52-W13-O41 & $84.0(9)$ & O16-W16-O59 & $95.0(8)$ & $\mathrm{O} 32-\mathrm{K} 1-\mathrm{O} 33$ & $63.6(5)$ \\
\hline O52-W13-O51 & $167.4(9)$ & O16-W16-O60 & $98.4(9)$ & $\mathrm{O} 32-\mathrm{K} 1-\mathrm{O} 45$ & $50.0(5)$ \\
\hline O59-W13-O41 & $85.0(7)$ & O56-W16-O46 & $83.4(7)$ & $\mathrm{O} 32-\mathrm{K} 1-\mathrm{O} 46$ & $85.3(5)$ \\
\hline O59-W13-O43 & $157.6(8)$ & O56-W16-O59 & $85.0(7)$ & $\mathrm{O} 33-\mathrm{K} 1-\mathrm{P} 2$ & $73.5(3)$ \\
\hline O59-W13-O51 & $86.5(7)$ & O58-W16-O46 & $84.4(7)$ & $\mathrm{O} 33-\mathrm{K} 1-\mathrm{O} 31$ & $117.9(5)$ \\
\hline O59-W13-O52 & $89.5(9)$ & O58-W16-O56 & $90.3(7)$ & $\mathrm{O} 33-\mathrm{K} 1-\mathrm{O} 45$ & $85.7(5)$ \\
\hline O14-W14-O44 & 177.1(11) & O58-W16-O59 & $166.9(7)$ & $\mathrm{O} 33-\mathrm{K} 1-\mathrm{O} 46$ & $49.8(5)$ \\
\hline O14-W14-O53 & $94.2(9)$ & O59-W16-O46 & $82.9(7)$ & $\mathrm{O} 44-\mathrm{K} 1-\mathrm{P} 2$ & $73.60(17)$ \\
\hline O14-W14-O57 & $97.7(8)$ & O60-W16-O46 & $83.5(8)$ & $\mathrm{O} 44-\mathrm{K} 1-\mathrm{O} 31$ & $49.4(7)$ \\
\hline O53-W14-O44 & $83.8(7)$ & O60-W16-O56 & $166.8(9)$ & $\mathrm{O} 44-\mathrm{K} 1-\mathrm{O} 32$ & $85.5(4)$ \\
\hline O53-W14-O57 & $89.6(7)$ & O60-W16-O58 & $89.2(9)$ & $\mathrm{O} 44-\mathrm{K} 1-\mathrm{O} 33$ & $147.0(4)$ \\
\hline O57-W14-O44 & $84.4(7)$ & O60-W16-O59 & $92.5(9)$ & $\mathrm{O} 44-\mathrm{K} 1-\mathrm{O} 45$ & $64.7(4)$ \\
\hline O15-W15-K6 & $42.3(7)$ & O61-Eu1-O1 & $141.9(14)$ & O44-K1-O46 & $120.6(5)$ \\
\hline
\end{tabular}




\begin{tabular}{|c|c|c|c|c|c|}
\hline O45-K1-P2 & $24.9(3)$ & K2-K3-O69B & $116.2(13)$ & O68B-K3-O71A & $126(2)$ \\
\hline $\mathrm{O} 45-\mathrm{K} 1-\mathrm{O} 31$ & $85.7(4)$ & $\mathrm{K} 2-\mathrm{K} 3-\mathrm{O} 72 \mathrm{~A}$ & $52.3(9)$ & O68B-K3-O69B & $59.6(19)$ \\
\hline $\mathrm{O} 46-\mathrm{K} 1-\mathrm{P} 2$ & $73.8(3)$ & $\mathrm{O} 3-\mathrm{K} 3-\mathrm{K} 2$ & $65.1(5)$ & O68B-K3-O72A & $134(2)$ \\
\hline O46-K1-O31 & $147.4(3)$ & $\mathrm{O} 3-\mathrm{K} 3-\mathrm{O} 70$ & $66.0(9)$ & O69B-K3-O72A & $165.9(16)$ \\
\hline O46-K1-O45 & $64.9(5)$ & O3-K3-O71A & $71.5(11)$ & W7-K4-W8 & $58.96(17)$ \\
\hline K3-K2-W3 & $72.9(2)$ & O3-K3-O68A & $112.2(12)$ & O7-K4-W7 & $25.9(4)$ \\
\hline $\mathrm{K} 3-\mathrm{K} 2-\mathrm{O} 2$ & $147.6(6)$ & O3-K3-O71B & $109.8(14)$ & O7-K4-W8 & $83.9(5)$ \\
\hline $\mathrm{K} 3-\mathrm{K} 2-\mathrm{O} 3$ & $51.3(5)$ & O3-K3-O72B & $114.7(12)$ & $\mathrm{O} 7-\mathrm{K} 4-\mathrm{O} 8$ & $105.9(7)$ \\
\hline K3-K2-O66 & $101.9(7)$ & O3-K3-O69B & $69.8(12)$ & O7-K4-O29 & $57.3(6)$ \\
\hline K3-K2-O67 & $87.5(6)$ & $\mathrm{O} 3-\mathrm{K} 3-\mathrm{O} 72 \mathrm{~A}$ & $96.5(14)$ & $\mathrm{O} 7-\mathrm{K} 4-\mathrm{O} 73$ & $99.8(9)$ \\
\hline $\mathrm{K} 3-\mathrm{K} 2-\mathrm{O} 72 \mathrm{~B}$ & $52.2(9)$ & O70-K3-O68A & $90.8(15)$ & O7-K4-O69B & $77.5(14)$ \\
\hline $\mathrm{O} 2-\mathrm{K} 2-\mathrm{W} 3$ & $77.4(4)$ & O70-K3-O71B & $50.5(16)$ & O8-K4-W7 & $82.3(4)$ \\
\hline $\mathrm{O} 2-\mathrm{K} 2-\mathrm{O} 66$ & $77.2(8)$ & O70-K3-O69B & $51.8(15)$ & O8-K4-W8 & $24.3(4)$ \\
\hline $\mathrm{O} 2-\mathrm{K} 2-\mathrm{O} 72 \mathrm{~B}$ & $156.2(12)$ & O70-K3-O72A & $127(2)$ & O29-K4-W7 & $31.4(4)$ \\
\hline O3-K2-W3 & $21.7(4)$ & $\mathrm{O} 71 \mathrm{~A}-\mathrm{K} 3-\mathrm{K} 2$ & $87.6(13)$ & O29-K4-W8 & $28.8(4)$ \\
\hline $\mathrm{O} 3-\mathrm{K} 2-\mathrm{O} 2$ & $98.9(6)$ & $\mathrm{O} 71 \mathrm{~A}-\mathrm{K} 3-\mathrm{O} 70$ & $68.5(15)$ & O29-K4-O8 & $53.1(6)$ \\
\hline O3-K2-O66 & $74.1(8)$ & O71A-K3-O68A & $155.9(18)$ & O73-K4-W7 & $83.7(7)$ \\
\hline $\mathrm{O} 3-\mathrm{K} 2-\mathrm{O} 67$ & $72.3(6)$ & O71A-K3-O71B & $105(2)$ & O73-K4-W8 & $69.1(7)$ \\
\hline $\mathrm{O} 3-\mathrm{K} 2-\mathrm{O} 72 \mathrm{~B}$ & $92.4(12)$ & O71A-K3-O69B & (17) & O73-K4-O8 & $75.9(9)$ \\
\hline O52-K2-W3 & $108.4(4)$ & $\mathrm{O} 71 \mathrm{~A}-\mathrm{K} 3-\mathrm{O} 72 \mathrm{~A}$ & $58(2)$ & O73-K4-O29 & $67.8(8)$ \\
\hline O66-K2-W3 & $66.3(6)$ & O69A-K3-K2 & $92.0(13)$ & O73-K4-O69B & $139.3(14)$ \\
\hline O67-K2-W3 & $67.2(5)$ & O69A-K3-O3 & $71.2(12)$ & O69B-K4-W7 & $77.0(12)$ \\
\hline $\mathrm{O} 67-\mathrm{K} 2-\mathrm{O} 2$ & $69.1(7)$ & O69A-K3-O70 & $81.0(15)$ & O69B-K4-W8 & $70.3(11)$ \\
\hline O67-K2-O66 & $127.0(8)$ & O69A-K3-O71A & $138.9(15)$ & O69B-K4-O8 & $66.3(13)$ \\
\hline O67-K2-O72B & $134.6(12)$ & O69A-K3-O68A & $41.7(16)$ & O69B-K4-O29 & $77.5(12)$ \\
\hline O72B-K2-W3 & $111.2(12)$ & O69A-K3-O71B & $72(2)$ & O11-K5-W12 & $81.2(5)$ \\
\hline O72B-K2-O66 & $86.0(13)$ & O69A-K3-O72B & $150.7(19)$ & $\mathrm{O} 11-\mathrm{K} 5-\mathrm{O} 50$ & $52.6(5)$ \\
\hline O72A-K2-W3 & $112(2)$ & O71B-K3-O68A & $51(2)$ & O11-K5-O75 & $62.9(7)$ \\
\hline $\mathrm{O} 72 \mathrm{~A}-\mathrm{K} 2-\mathrm{K} 3$ & $67.7(11)$ & $\mathrm{O} 72 \mathrm{~B}-\mathrm{K} 3-\mathrm{K} 2$ & $67.7(11)$ & O11-K5-O77 & $73.7(5)$ \\
\hline $\mathrm{O} 72 \mathrm{~A}-\mathrm{K} 2-\mathrm{O} 2$ & $137.7(14)$ & O72B-K3-O70 & $128.1(17)$ & O11-K5-O76A & $78.1(10)$ \\
\hline $\mathrm{O} 72 \mathrm{~A}-\mathrm{K} 2-\mathrm{O} 3$ & $97.8(18)$ & O72B-K3-O71A & $64.1(19)$ & O12-K5-W12 & $22.8(4)$ \\
\hline $\mathrm{O} 72 \mathrm{~A}-\mathrm{K} 2-\mathrm{O} 66$ & $71(2)$ & O72B-K3-O68A & $128.0(18)$ & O12-K5-O11 & $103.8(6)$ \\
\hline $\mathrm{O} 72 \mathrm{~A}-\mathrm{K} 2-\mathrm{O} 67$ & $153.2(13)$ & O72B-K3-O71B & $126.0(17)$ & O12-K5-O50 & $52.1(5)$ \\
\hline $\mathrm{K} 2-\mathrm{K} 3-\mathrm{O} 70$ & $130.2(8)$ & O68B-K3-K2 & $145.4(18)$ & O12-K5-O75 & $154.1(10)$ \\
\hline $\mathrm{K} 2-\mathrm{K} 3-\mathrm{O} 68 \mathrm{~A}$ & $115.8(13)$ & O68B-K3-O3 & $128.9(16)$ & O12-K5-O77 & $72.6(5)$ \\
\hline $\mathrm{K} 2-\mathrm{K} 3-\mathrm{O} 71 \mathrm{~B}$ & $164.2(16)$ & O68B-K3-O70 & $77.0(18)$ & O12-K5-O76A & $73.7(10)$ \\
\hline
\end{tabular}




\begin{tabular}{|c|c|c|c|c|c|}
\hline O50-K5-W12 & $29.4(3)$ & O77-K6-W15 & $78.0(3)$ & O35-P3-O31 & $109.6(9)$ \\
\hline O50-K5-O584 & $137.6(6)$ & O77-K6-O11 & $81.2(5)$ & O44-P3-K1 & $53.7(10)$ \\
\hline O50-K5-O75 & $108.0(8)$ & O77-K6-O15 & $80.7(5)$ & O44-P3-O31 & $109.4(14)$ \\
\hline O50-K5-O77 & $70.0(4)$ & O77-K6-O55 & $68.5(5)$ & O44-P3-O35 & $110.2(9)$ \\
\hline O74-K5-W12 & $142.5(10)$ & O77-K6-O79 & $135.7(8)$ & W1-O1-Eu1 & 177.1(16) \\
\hline O74-K5-O11 & $130.3(10)$ & O78-K6-W15 & $86.4(9)$ & $\mathrm{W} 2-\mathrm{O} 2-\mathrm{K} 2$ & 119.9(9) \\
\hline O74-K5-O12 & $122.7(10)$ & O78-K6-O11 & $120.0(12)$ & W3-O3-K2 & $121.5(10)$ \\
\hline O74-K5-O50 & $156.3(10)$ & O78-K6-O15 & $75.7(9)$ & W3-O3-K3 & $172.8(11)$ \\
\hline O74-K5-O75 & $67.5(10)$ & O78-K6-O55 & $107.9(10)$ & $\mathrm{K} 3-\mathrm{O} 3-\mathrm{K} 2$ & $63.6(5)$ \\
\hline O74-K5-O77 & 133.1(9) & O78-K6-O77 & $152.6(10)$ & W7-O7-K4 & $110.2(9)$ \\
\hline O74-K5-O76A & $97.8(12)$ & O78-K6-O79 & $71.1(12)$ & W8-O8-K4 & 107.0(9) \\
\hline O75-K5-W12 & $135.8(8)$ & O79-K6-W15 & $111.9(8)$ & $\mathrm{W} 11-\mathrm{O} 11-\mathrm{K} 5$ & 116.3(9) \\
\hline O75-K5-O77 & $119.8(8)$ & O79-K6-O11 & $58.1(8)$ & W11-O11-K6 & 101.5(9) \\
\hline O77-K5-W12 & $67.97(19)$ & O79-K6-O55 & $97.3(8)$ & K5-O11-K6 & $93.2(6)$ \\
\hline O76A-K5-W12 & $65.9(8)$ & O33-P1-K1 & $54.4(7)$ & $\mathrm{W} 12-\mathrm{O} 12-\mathrm{K} 5$ & 117.0(9) \\
\hline O76A-K5-O50 & $58.7(9)$ & O33-P1-O41 & 109.1(10) & W15-O15-K6 & $112.4(10)$ \\
\hline O76A-K5-O75 & $81.5(13)$ & $\mathrm{O} 40-\mathrm{P} 1-\mathrm{K} 1$ & $125.2(7)$ & W4-O17-W1 & $150.3(11)$ \\
\hline O76A-K5-O77 & $128.6(8)$ & O40-P1-O33 & $109.9(10)$ & W1-O18-W2 & $162.4(11)$ \\
\hline O76B-K5-W12 & $77.6(16)$ & O40-P1-O41 & $110.9(10)$ & W3-O19-W2 & $161.4(10)$ \\
\hline O76B-K5-O11 & $97.9(18)$ & O41-P1-K1 & $123.9(7)$ & W8-O21-W3 & $149.3(10)$ \\
\hline O76B-K5-O12 & $77.2(17)$ & O46-P1-K1 & $54.8(7)$ & $\mathrm{W} 5-\mathrm{O} 22-\mathrm{W} 2$ & $152.4(10)$ \\
\hline O76B-K5-O50 & $78.2(17)$ & O46-P1-O33 & $109.2(10)$ & W6-O23-W2 & $150.8(10)$ \\
\hline O76B-K5-O74 & $78.2(19)$ & $\mathrm{O} 46-\mathrm{P} 1-\mathrm{O} 40$ & $108.9(10)$ & $\mathrm{W} 7-\mathrm{O} 24-\mathrm{W} 3$ & $150.0(9)$ \\
\hline O76B-K5-O75 & $82.6(19)$ & O46-P1-O41 & $108.7(9)$ & W5-O26-W4 & $160.1(10)$ \\
\hline O76B-K5-O77 & $145.4(16)$ & $\mathrm{O} 32-\mathrm{P} 2-\mathrm{K} 1$ & $54.6(7)$ & W5-O27-W6 & $149.7(11)$ \\
\hline O11-K6-W15 & $84.9(5)$ & $\mathrm{O} 32-\mathrm{P} 2-\mathrm{O} 37$ & $111.1(10)$ & W6-O28-W7 & $160.3(10)$ \\
\hline O15-K6-W15 & $25.3(4)$ & O37-P2-K1 & $125.9(7)$ & W7-O29-W8 & $148.9(10)$ \\
\hline O15-K6-O11 & $110.2(7)$ & O38-P2-K1 & $125.5(7)$ & W7-O29-K4 & $96.2(7)$ \\
\hline O15-K6-O55 & $55.7(6)$ & $\mathrm{O} 38-\mathrm{P} 2-\mathrm{O} 32$ & $109.5(10)$ & W8-O29-K4 & $105.1(7)$ \\
\hline O15-K6-O79 & $126.9(9)$ & $\mathrm{O} 38-\mathrm{P} 2-\mathrm{O} 37$ & $108.6(10)$ & W1-O31-K1 & $129.7(10)$ \\
\hline O164-K6-W15 & $141.8(6)$ & $\mathrm{O} 45-\mathrm{P} 2-\mathrm{K} 1$ & $55.5(7)$ & P3-O31-W1 & $131.1(14)$ \\
\hline O164-K6-O11 & $62.7(6)$ & $\mathrm{O} 45-\mathrm{P} 2-\mathrm{O} 32$ & $110.1(10)$ & P3-O31-K1 & $99.1(11)$ \\
\hline O164-K6-O55 & $111.5(6)$ & $\mathrm{O} 45-\mathrm{P} 2-\mathrm{O} 37$ & $108.5(10)$ & W2-O32-K1 & $129.4(7)$ \\
\hline O164-K6-O79 & $69.1(8)$ & $\mathrm{O} 45-\mathrm{P} 2-\mathrm{O} 38$ & $109.0(10)$ & $\mathrm{P} 2-\mathrm{O} 32-\mathrm{W} 2$ & $130.2(10)$ \\
\hline O55-K6-W15 & $31.1(4)$ & O31-P3-K1 & $55.7(9)$ & $\mathrm{P} 2-\mathrm{O} 32-\mathrm{K} 1$ & $100.3(8)$ \\
\hline O55-K6-O11 & $54.9(6)$ & O35-P3-K1 & $126.1(7)$ & W3-O33-K1 & $129.2(7)$ \\
\hline
\end{tabular}




\begin{tabular}{llllll}
\hline P1-O33-W3 & $130.3(10)$ & W8-O41-W13 & $94.5(6)$ & W11-O50-K5 & $93.1(6)$ \\
P1-O33-K1 & $100.5(8)$ & P1-O41-W8 & $124.8(9)$ & W12-O50-W11 & $161.3(10)$ \\
W9-O34-W4 & $118.6(9)$ & P1-O41-W13 & $124.2(10)$ & W12-O50-K5 & $93.2(7)$ \\
W4-O35-W9 & $94.4(6)$ & W12-O42-W7 & $117.6(8)$ & W13-O51-W12 & $147.1(9)$ \\
P3-O35-W4 & $127.0(11)$ & W13-O43-W8 & $119.6(10)$ & W9-O53-W14 & $148.4(11)$ \\
P3-O35-W9 & $126.3(11)$ & W14-O44-K1 & $126.7(10)$ & W10-O54-W15 & $150.0(10)$ \\
W5-O36-W10 & $121.1(9)$ & P3-O44-W14 & $131.2(14)$ & W11-O55-W15 & $150.0(10)$ \\
W10-O37-W5 & $94.2(6)$ & P3-O44-K1 & $102.0(12)$ & W11-O55-K6 & $100.2(7)$ \\
P2-O37-W5 & $125.6(11)$ & W15-O45-K1 & $127.2(7)$ & W15-O55-K6 & $95.8(7)$ \\
P2-O37-W10 & $126.5(10)$ & P2-O45-W15 & $133.2(10)$ & W12-O56-W16 & $150.4(10)$ \\
W6-O38-W11 & $93.7(6)$ & P2-O45-K1 & $99.6(8)$ & W15-O57-W14 & $160.7(10)$ \\
P2-O38-W6 & $126.2(10)$ & W16-O46-K1 & $127.4(7)$ & W15-O58-W16 & $162.6(10)$ \\
P2-O38-W11 & $127.0(11)$ & P1-O46-W16 & $132.1(10)$ & W13-O59-W16 & $151.3(10)$ \\
W6-O39-W11 & $119.5(9)$ & P1-O46-K1 & $100.5(8)$ & K6-O77-K5 & $90.7(3)$ \\
W12-O40-W7 & $93.7(6)$ & W91-O47-W9 & $149.8(16)$ & K3-O72B-K2 & $60.0(9)$ \\
P1-O40-W7 & $125.4(9)$ & W9-O48-W10 & $160.2(11)$ & K3-O69B-K4 & $173(3)$ \\
P1-O40-W12 & $126.3(10)$ & W10-O49-W11 & $146.9(10)$ & K2-O72A-K3 & $60.0(9)$ \\
\hline
\end{tabular}


Atomic numbering scheme of compound I
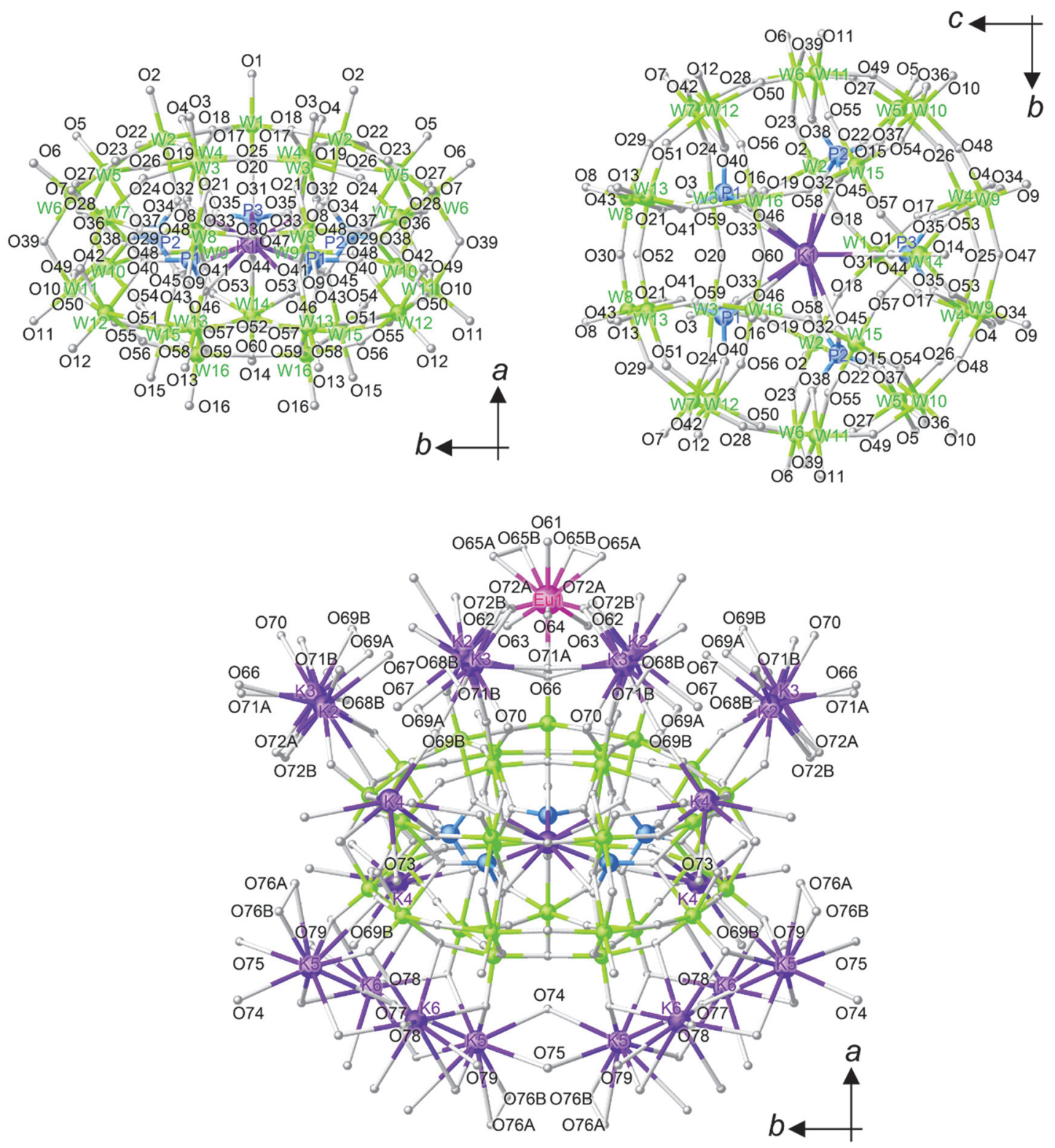
Table S4. Cell parameters of II calculated by the Pawley fitting of PXRD.

\begin{tabular}{|c|c|c|c|c|c|}
\hline & Under $\mathrm{N}_{2}$ & RH $30 \%$ & RH $50 \%$ & RH 75\% & RH $100 \%$ \\
\hline Crystal system & & & triclinic & & \\
\hline Space group & & & $P-1$ & & \\
\hline$a / \AA$ & $19.556(55)$ & $19.53(13)$ & $19.52(18)$ & $19.605(66)$ & $19.695(75)$ \\
\hline$b / \AA$ & $23.154(55)$ & $23.13(19)$ & $23.17(19)$ & $23.270(76)$ & $23.28(11)$ \\
\hline$c / \AA$ & $27.926(80)$ & $28.08(23)$ & $28.20(32)$ & $27.74(11)$ & $27.85(11)$ \\
\hline$\alpha /^{\circ}$ & $92.809(91)$ & $92.27(36)$ & $92.52(32)$ & $92.05(16)$ & $92.95(23)$ \\
\hline$\beta / \circ$ & $91.48(20)$ & $92.07(64)$ & $91.34(57)$ & $91.85(26)$ & $91.86(19)$ \\
\hline$\gamma / \circ$ & $92.64(10)$ & $91.93(33)$ & $92.03(33)$ & $92.29(15)$ & $91.85(15)$ \\
\hline Volume/ $\AA^{3}$ & $12611(59)$ & $12660(170)$ & $12730(210)$ & $12631(79)$ & $12742(91)$ \\
\hline
\end{tabular}


Table S5. Comparison with representative proton conductors.

\begin{tabular}{|c|c|c|c|c|}
\hline Compounds & $\begin{array}{l}\text { Conductivity } \\
(\sigma)\left[\mathrm{S} \mathrm{cm}^{-1}\right]\end{array}$ & $\begin{array}{c}\text { Activation } \\
\text { energy }\left(E_{\mathrm{a}}\right)[\mathrm{eV}]\end{array}$ & Conditions & Ref. \\
\hline $\mathrm{H}_{3} \mathrm{PO}_{4} @ \mathrm{NKCOF}-1$ & $1.1 \times 10^{-1}$ & 0.14 & $\begin{array}{c}353 \mathrm{~K}, \\
98 \% \mathrm{RH}\end{array}$ & {$[1]$} \\
\hline KNaLi-1 & $4.5 \times 10^{-2}$ & 0.23 & $\begin{array}{c}358 \mathrm{~K} \\
70 \% \mathrm{RH}\end{array}$ & {$[2]$} \\
\hline MOF-74 (Mg) & $2.6 \times 10^{-2}$ & ca. 0.1 & $\begin{array}{c}298 \mathrm{~K} \\
95 \% \mathrm{RH}\end{array}$ & {$[3]$} \\
\hline TETA@3 (HPWs@MIL-101) & $1.5 \times 10^{-2}$ & 0.22 & $\begin{array}{c}353 \mathrm{~K} \\
100 \% \mathrm{RH}\end{array}$ & {$[4]$} \\
\hline$\left[\mathrm{Ln}_{27} \mathrm{Ge}_{10} \mathrm{~W}_{106} \mathrm{O}_{406}(\mathrm{OH})_{4}\left(\mathrm{H}_{2} \mathrm{O}\right)_{24}\right]^{59-}$ & $1.5 \times 10^{-2}$ & 0.42 & $\begin{array}{c}358 \mathrm{~K}, \\
98 \% \mathrm{RH}\end{array}$ & {$[5]$} \\
\hline$\left[\mathrm{Ln}_{3}\left(\mathrm{H}_{2} \mathrm{O}\right)_{22}\right]\left[\mathrm{P}_{2} \mathrm{~W}_{15} \mathrm{Ta}_{3} \mathrm{O}_{62}\right] \cdot n \mathrm{H}_{2} \mathrm{O}$ & $1.3 \times 10^{-2}$ & 0.36 & $\begin{array}{c}368 \mathrm{~K} \\
98 \% \mathrm{RH}\end{array}$ & {$[6]$} \\
\hline Im-Fe-MOF & $1.2 \times 10^{-2}$ & 0.44 & $\begin{array}{c}333 \mathrm{~K}, \\
98 \% \mathrm{RH}\end{array}$ & {$[7]$} \\
\hline II & $1.2 \times 10^{-2}$ & 0.48 & $\begin{array}{c}368 \mathrm{~K}, \\
90 \% \mathrm{RH}\end{array}$ & $\begin{array}{l}\text { This } \\
\text { work }\end{array}$ \\
\hline $\begin{array}{l}\mathrm{K}_{5} \mathrm{H}_{7}\left[\mathrm{Bi}\left(\mathrm{H}_{2} \mathrm{O}\right) \mathrm{P}_{5} \mathrm{~W}_{30} \mathrm{O}_{110}\right] \\
\cdot 0.11 \mathrm{PAA} 3000 \cdot 16 \mathrm{H}_{2} \mathrm{O}\end{array}$ & $9.7 \times 10^{-3}$ & 0.24 & $\begin{array}{c}368 \mathrm{~K} \\
75 \% \mathrm{RH}\end{array}$ & {$[8]$} \\
\hline La-PCMOF-5 & $6.0 \times 10^{-3}$ & 0.17 & $\begin{array}{c}358 \mathrm{~K}, \\
95 \% \mathrm{RH}\end{array}$ & {$[9]$} \\
\hline EB-COF: PW12 & $3.3 \times 10^{-3}$ & $\begin{array}{c}0.24 \\
\text { (ca. } 53 \% \mathrm{RH})\end{array}$ & $\begin{array}{c}298 \mathrm{~K} \\
97 \% \mathrm{RH}\end{array}$ & {$[10]$} \\
\hline $\begin{array}{l}\mathrm{Na}_{16}\left(\mathrm{NH}_{4}\right)_{10} \\
\mathrm{H}_{8}\left\{\left[\mathrm{~W}_{14} \mathrm{Ce}^{\mathrm{IV}}{ }_{6} \mathrm{O}_{61}\right]\left(\mathrm{W}_{3} \mathrm{Bi}_{6} \mathrm{Ce}^{\mathrm{III}}{ }_{3}\left(\mathrm{H}_{2} \mathrm{O}\right)_{3}\right.\right. \\
\left.\left.\left.\mathrm{O}_{14}\right]-\left[\alpha-\mathrm{BiW}_{9} \mathrm{O}_{33}\right]_{3}\right)_{2}\right\} \cdot \text { ca. } 38 \mathrm{H}_{2} \mathrm{O}\end{array}$ & $2.4 \times 10^{-3}$ & 0.68 & $\begin{array}{c}298 \mathrm{~K} \\
90 \% \mathrm{RH}\end{array}$ & {$[11]$} \\
\hline $\begin{array}{l}{\left[\mathrm{Eu}_{20}(\mathrm{PDC})_{12}\left(\mathrm{SO}_{4}\right)_{12}\left(\mu_{2}-\mathrm{OH}\right)_{3}\left(\mu_{2^{-}}\right.\right.} \\
\left.\left.\mathrm{H}_{2} \mathrm{O}\right)_{3}\left(\mathrm{H}_{2} \mathrm{O}\right)_{36}\right]_{n}(\mathrm{Eu}-\mathrm{SCP})\end{array}$ & $1.2 \times 10^{-3}$ & 0.19 & $\begin{array}{c}298 \mathrm{~K} \\
97 \% \mathrm{RH}\end{array}$ & {$[12]$} \\
\hline
\end{tabular}




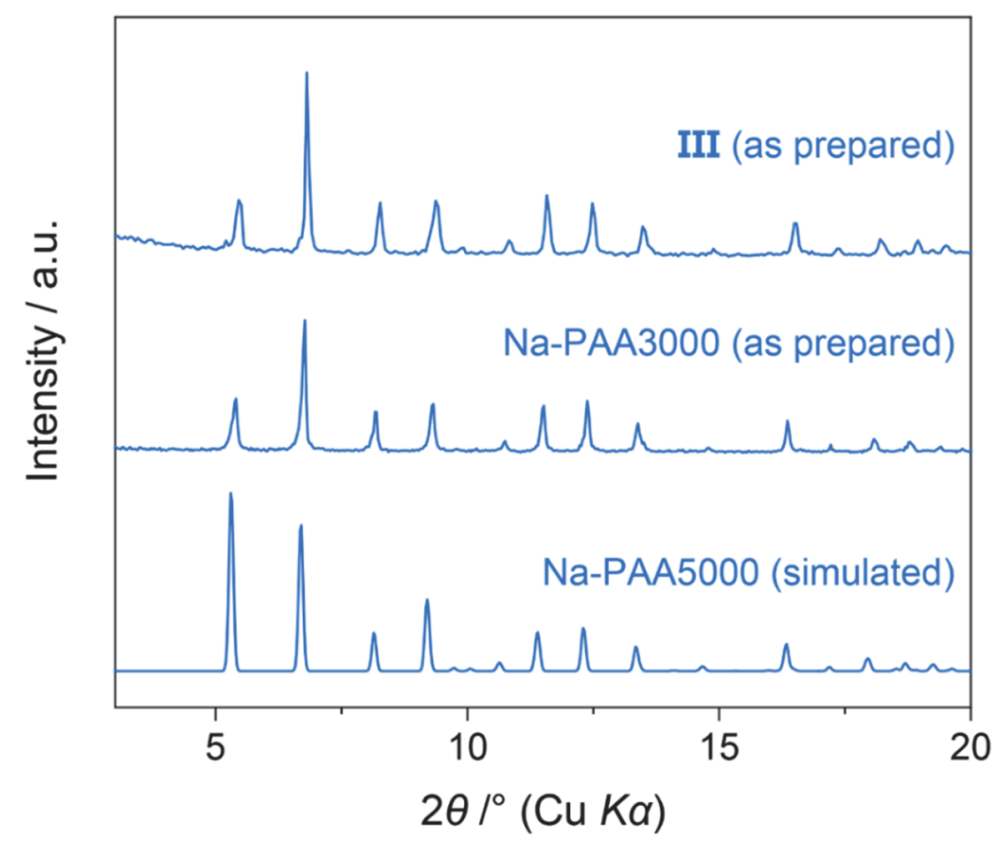

Figure S1. PXRD patterns of III (top) and crystalline composites composed of $\left[\mathrm{P}_{5} \mathrm{~W}_{30} \mathrm{O}_{110} \mathrm{Na}\left(\mathrm{H}_{2} \mathrm{O}\right)\right]^{14-}$ and PAAs (middle and bottom). ${ }^{13}$ Reprinted in part with permission from ref. 13. Copyright 2020 Elsevier.

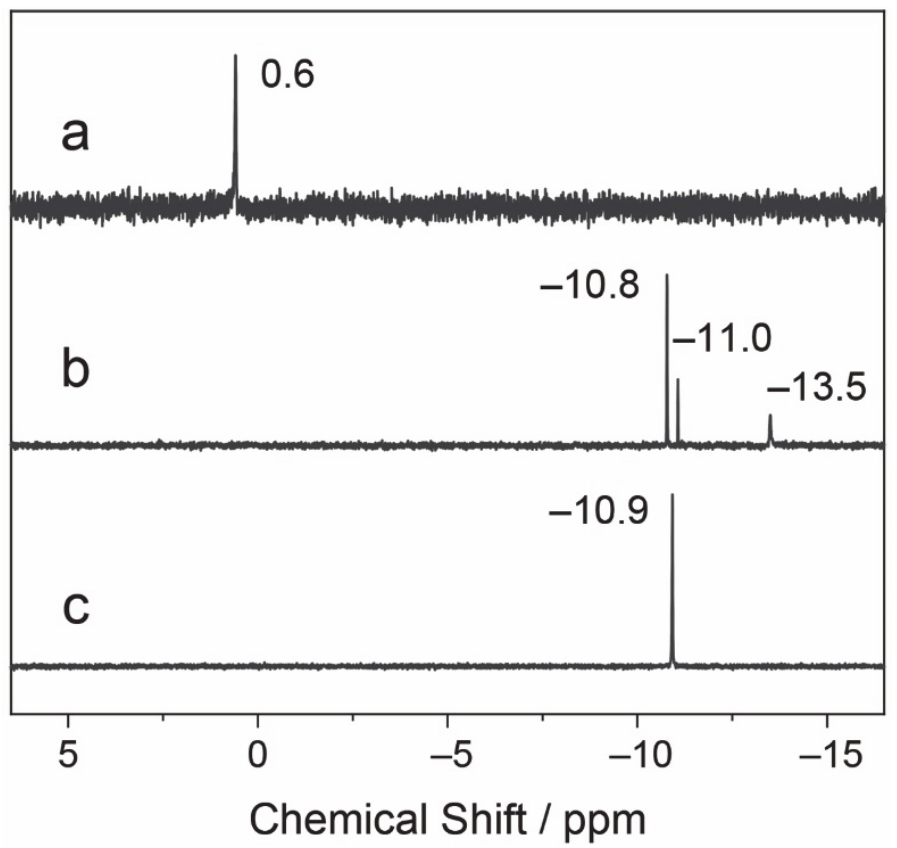

Figure S2. ${ }^{31} \mathrm{P}-\mathrm{NMR}$ spectra of (a) $\mathrm{K}_{12}\left[\mathrm{P}_{5} \mathrm{~W}_{30} \mathrm{O}_{110} \mathrm{Eu}\left(\mathrm{H}_{2} \mathrm{O}\right)\right] \cdot 24 \mathrm{H}_{2} \mathrm{O}$, (b) the solid obtained after heating (a), and (c) $\mathbf{I}$ in $\mathrm{D}_{2} \mathrm{O}$. The assignment of the minor signal at $-13.5 \mathrm{ppm}$ is unknown at this moment. 


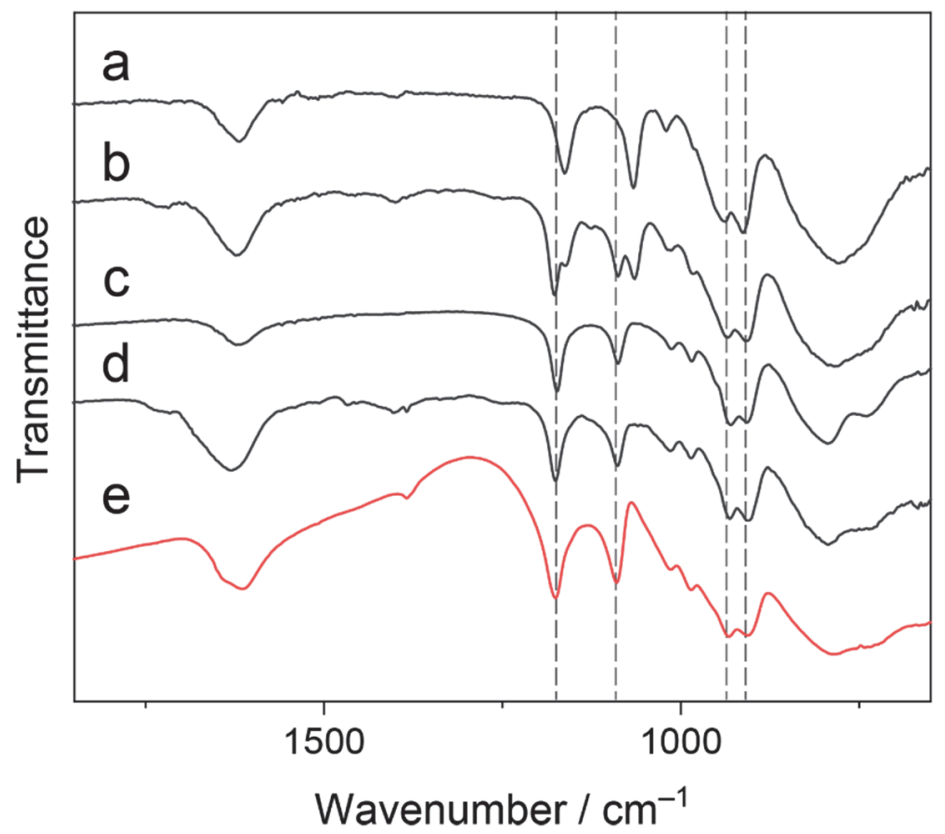

Figure S3. IR spectra of (a) $\mathrm{K}_{12}\left[\mathrm{P}_{5} \mathrm{~W}_{30} \mathrm{O}_{110} \mathrm{Eu}\left(\mathrm{H}_{2} \mathrm{O}\right)\right] \cdot 24 \mathrm{H}_{2} \mathrm{O}$, (b) the solid after heating $\mathrm{K}_{12}\left[\mathrm{P}_{5} \mathrm{~W}_{30} \mathrm{O}_{110} \mathrm{Eu}\left(\mathrm{H}_{2} \mathrm{O}\right)\right] \cdot 24 \mathrm{H}_{2} \mathrm{O}$, (c) $\mathrm{K}_{14}\left[\mathrm{P}_{5} \mathrm{~W}_{30} \mathrm{O}_{110} \mathrm{~K}\right] \cdot 24 \mathrm{H}_{2} \mathrm{O}$, (d) I, and (e) II. Broken lines indicate the positions of characteristic absorption bands of $\mathbf{I}$. 


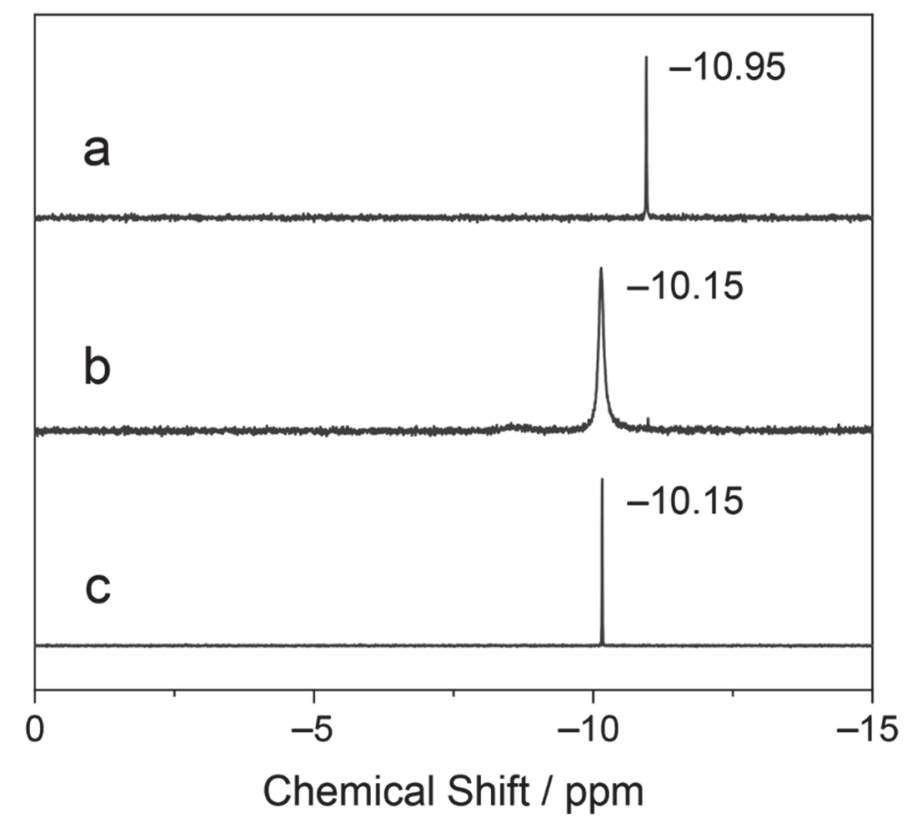

Figure S4. ${ }^{31} \mathrm{P}$ NMR spectra of (a) I, (b) $\mathrm{H}_{14}\left[\mathrm{P}_{5} \mathrm{~W}_{30} \mathrm{O}_{110} \mathrm{~K}\right]$, and (c) after neutralization of (b) with $\mathrm{K}_{2} \mathrm{CO}_{3}$ in $\mathrm{D}_{2} \mathrm{O}$. The attached $\mathrm{Eu}^{3+}$ in (a) $\mathrm{I}$ can be exchanged with protons $\left(\mathrm{H}^{+}\right)$by passing a solution through an ion exchange resin in its acid form to give (b) the protonic form of $\left[\mathrm{P}_{5} \mathrm{~W}_{30} \mathrm{O}_{110} \mathrm{~K}\right]^{14}$, which was confirmed by the presence of a broad signal in the ${ }^{31} \mathrm{P}$ NMR spectrum. ${ }^{2}$ Subsequent neutralization with $\mathrm{K}_{2} \mathrm{CO}_{3}$ produced the $(\mathrm{c}) \mathrm{K}^{+}$salt which was characterized by its sharp ${ }^{31} \mathrm{P}$ NMR signal. $^{14}$ 

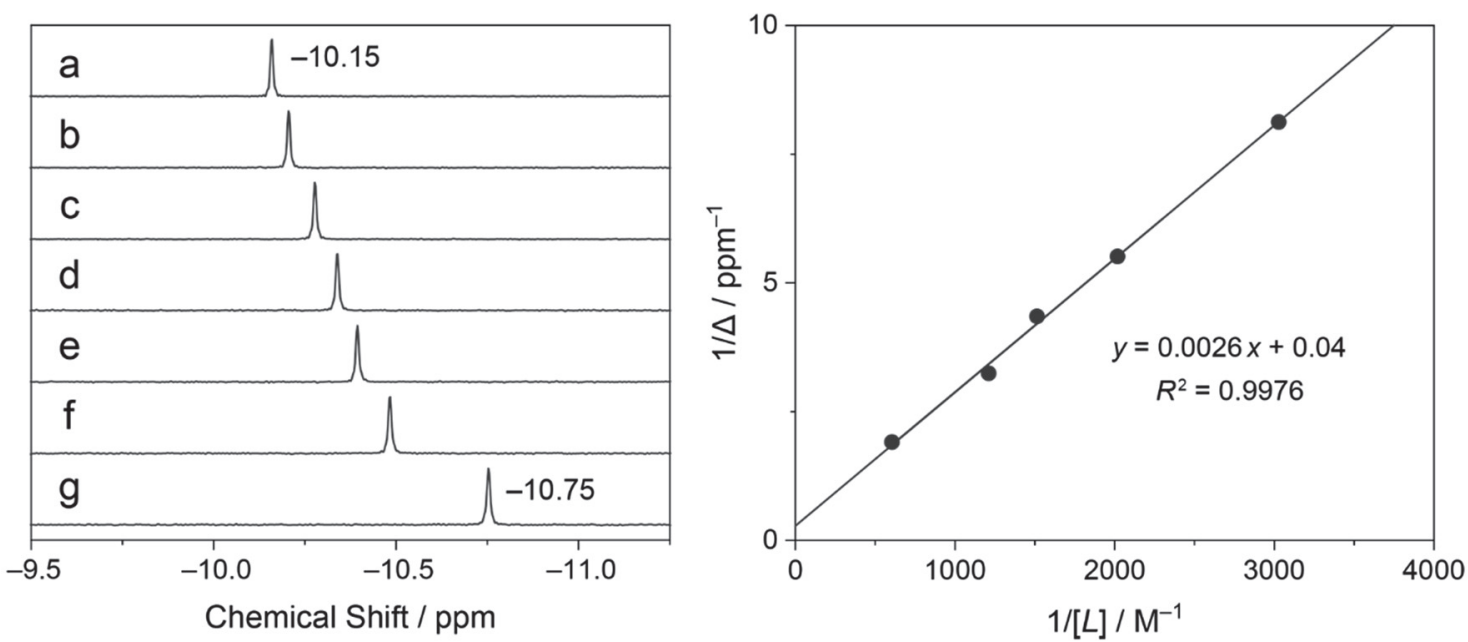

Figure S5. Left: ${ }^{31} \mathrm{P}$ NMR spectra of (a) $\mathrm{K}_{14}\left[\mathrm{P}_{5} \mathrm{~W}_{30} \mathrm{O}_{110} \mathrm{~K}\right]$ in $\mathrm{D}_{2} \mathrm{O}$ and after adding (b) 0.1 , (c) 0.2 , (d) 0.3 , (e) 0.4 , (f) 0.5 , and (g) 1.0 equivalent of $\mathrm{Eu}\left(\mathrm{NO}_{3}\right)_{3}$ at $293 \mathrm{~K}$. Right: plot of $1 / \Delta$ against $1 /[L]$ (solid circles) and the regression line (solid line) to estimate the binding constant $K$ at 293 $\mathrm{K}$.

As shown in Figure S5 (left), the ${ }^{31} \mathrm{P}$ NMR signal at $-10.15 \mathrm{ppm}$ for $\left[\mathrm{P}_{5} \mathrm{~W}_{30} \mathrm{O}_{110} \mathrm{~K}\right]^{14-}$ gradually shifted to the negative direction by the addition of $\mathrm{Eu}^{3+}\left(\mathrm{Eu}\left(\mathrm{NO}_{3}\right)_{3}\right)$ and appeared at $-10.75 \mathrm{ppm}$ by the addition of one equivalent of $\mathrm{Eu}^{3+}$. The presence of a single peak indicates that the addition and removal of $\mathrm{Eu}^{3+}$ (equation 1) is faster than the NMR timescale. Assuming that one $\mathrm{Eu}^{3+}$ interacts with one $\left[\mathrm{P}_{5} \mathrm{~W}_{30} \mathrm{O}_{110} \mathrm{~K}\right]^{14-}$ (equation 1), the binding constant $\mathrm{K}$ (equation 2) at $293 \mathrm{~K}$ was estimated by using equation 3 , where $\Delta$ is the observed chemical shift - chemical shift of $\left[\mathrm{P}_{5} \mathrm{~W}_{30} \mathrm{O}_{110} \mathrm{~K}\right]^{14-}, \Delta_{11}$ is the chemical shift difference between $\left[\mathrm{P}_{5} \mathrm{~W}_{30} \mathrm{O}_{110} \mathrm{~K}\right]^{14-}$ and $\mathrm{Eu}\left[\mathrm{P}_{5} \mathrm{~W}_{30} \mathrm{O}_{110} \mathrm{~K}\right]^{11-}$, and $[\mathrm{L}]$ is the concentration of $\mathrm{Eu}^{3+} .{ }^{15}$ The binding constant $\mathrm{K}(293 \mathrm{~K})$ was estimated as $15.4\left[\mathrm{M}^{-1}\right]$ by the plot of $1 / \Delta$ against $1 /[\mathrm{L}]$ shown in Figure S5 (right).

$$
\begin{array}{lll}
{\left[\mathrm{P}_{5} \mathrm{~W}_{30} \mathrm{O}_{110} \mathrm{~K}\right]^{14-}+\mathrm{Eu}^{3+} \rightleftarrows \mathrm{Eu}\left[\mathrm{P}_{5} \mathrm{~W}_{30} \mathrm{O}_{110} \mathrm{~K}\right]^{11-}} & \text { (equation 1) } \\
K=\left[\mathrm{Eu}\left[\mathrm{P}_{5} \mathrm{~W}_{30} \mathrm{O}_{110} \mathrm{~K}\right]^{11-}\right] /\left[\left[\mathrm{P}_{5} \mathrm{~W}_{30} \mathrm{O}_{110} \mathrm{~K}\right]^{14-}\right]\left[\mathrm{Eu}^{3+}\right] & {\left[\mathrm{M}^{-1}\right]} & \text { (equation 2) } \\
1 / \Delta=1 / \Delta_{11} K[L]+1 / \Delta_{11} &
\end{array}
$$



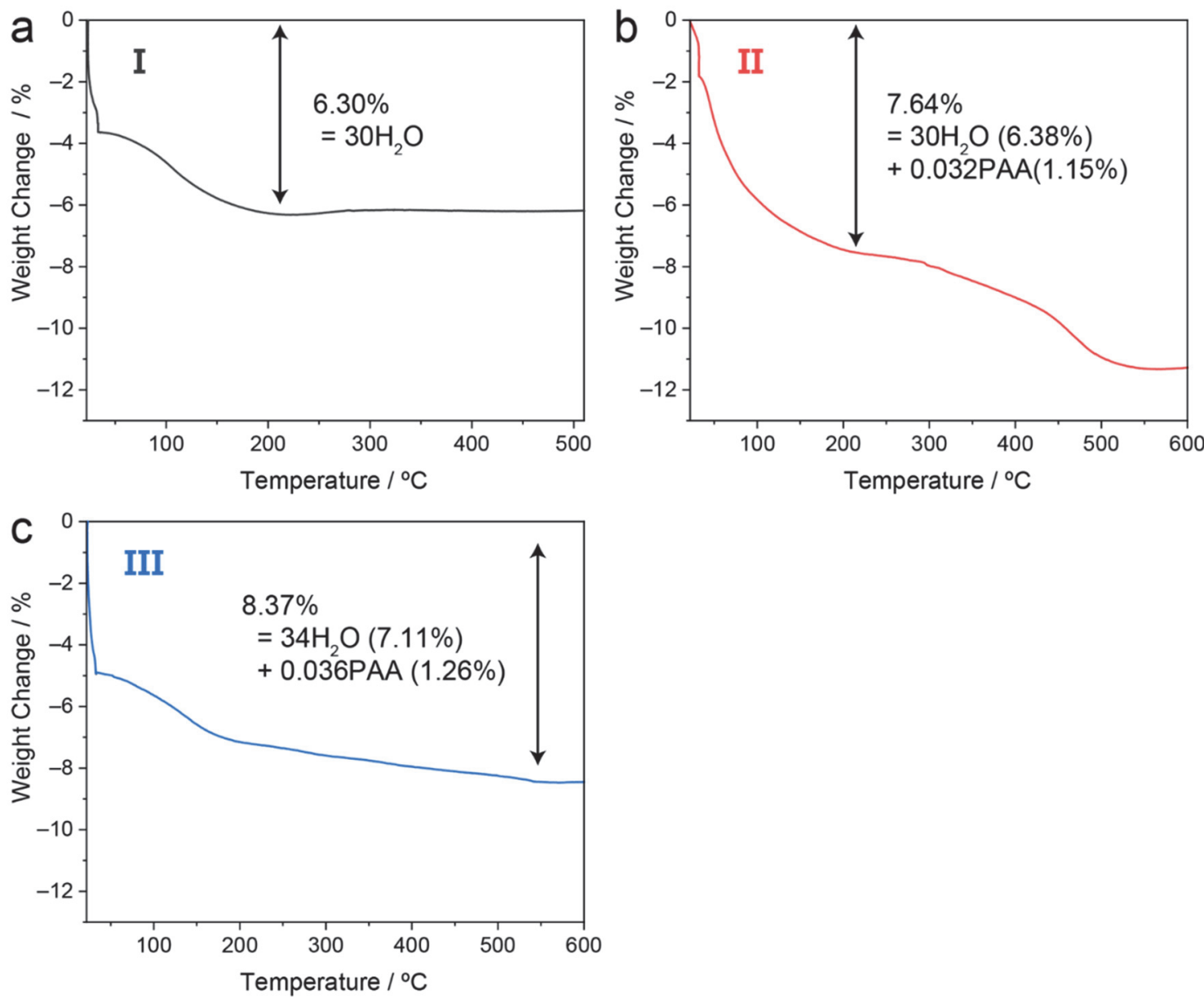

Figure S6. TG of (a) I, (b) II, and (c) III. 


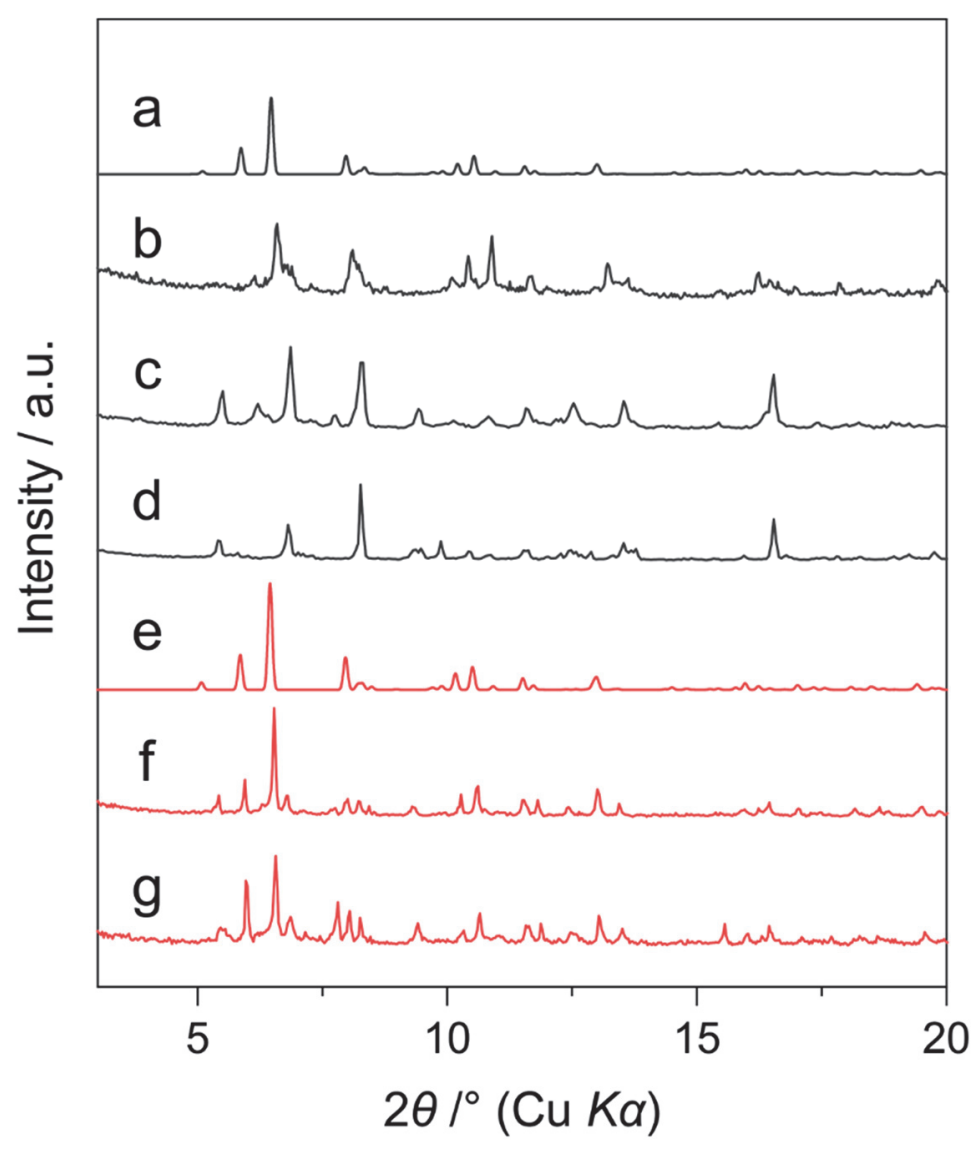

Figure S7. PXRD patterns of (a) I (calc), (b) I, (c) I (under saturated water vapor condition), (d) $\mathrm{K}_{14}\left[\mathrm{P}_{5} \mathrm{~W}_{30} \mathrm{O}_{110} \mathrm{~K}\right]$, (e) II (calc), (f) II, and (g) II (under saturated water vapor condition).
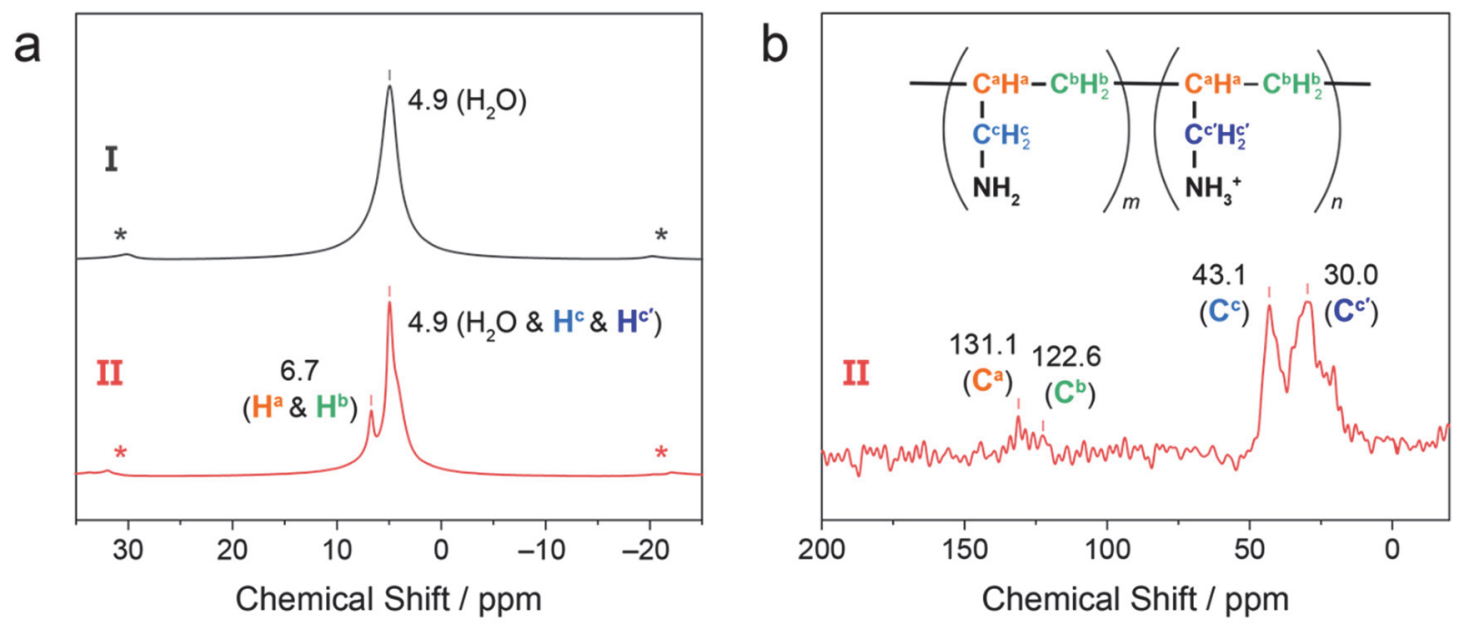

Figure S8. (a) ${ }^{1} \mathrm{H}$ MAS NMR spectra (MAS $=10 \mathrm{kHz}$ ) of I (black) and II (red). (b) ${ }^{13} \mathrm{C} \mathrm{CP} \mathrm{MAS}$ NMR spectrum (MAS $=5 \mathrm{kHz}$ ) of II. Asterisks in (a) denotes the spinning side bands. 


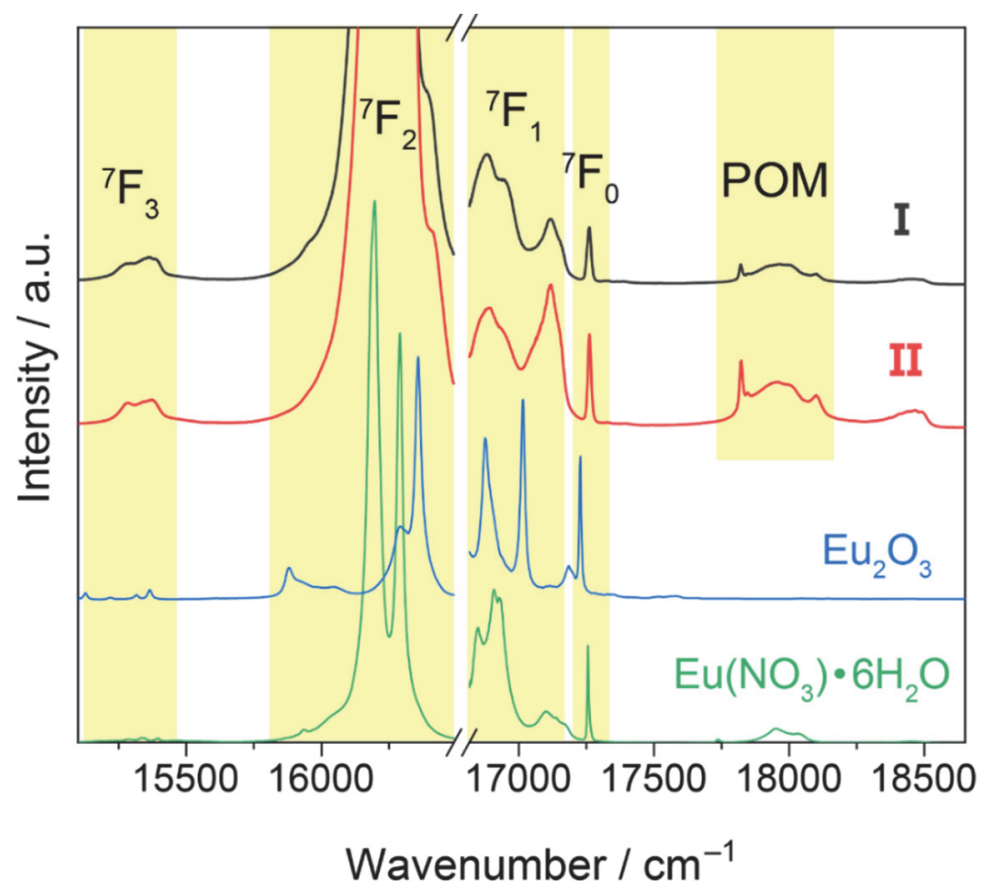

Figure S9. Raman/luminescence emission spectra of I (black) and II (red), together with those of $\mathrm{Eu}_{2} \mathrm{O}_{3}$ (blue) and $\mathrm{Eu}\left(\mathrm{NO}_{3}\right) \cdot 6 \mathrm{H}_{2} \mathrm{O}$ (green) as references. The signals around $18000 \mathrm{~cm}^{-1}$ in $\mathbf{I}$ and II are the characteristic Raman bands of the Preyssler-type POM.
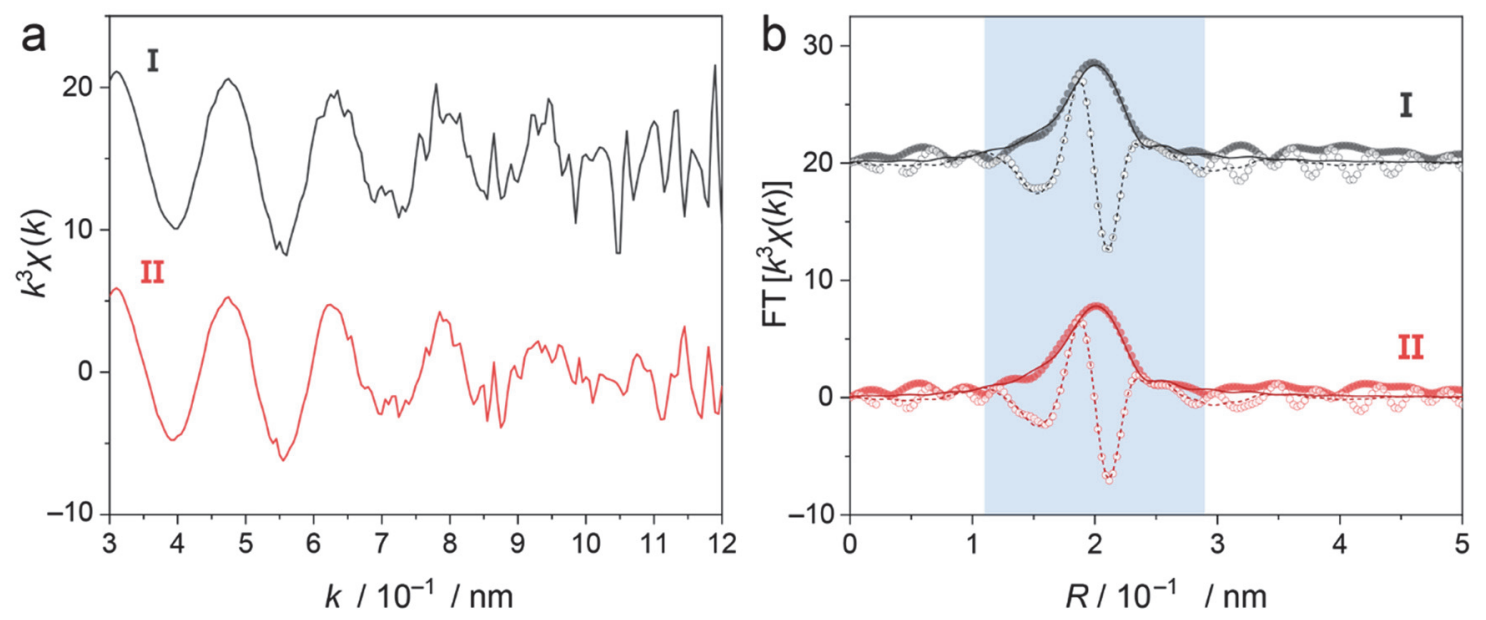

Figure S10. (a) $k^{3}$-Weighed Eu LIII-edge EXAFS oscillations and (b) their Fourier transforms $(k$ $=30-120 \mathrm{~nm}^{-1}, R=0.11-0.29 \mathrm{~nm}$ ) for I (black) and II (red). Closed and open circles in (b) show modulus and imaginary part of the FT for observed data, respectively, while solid and dashed lines show modulus and imaginary part of the FT for fitted data, respectively. The fitting range is highlighted in blue. 

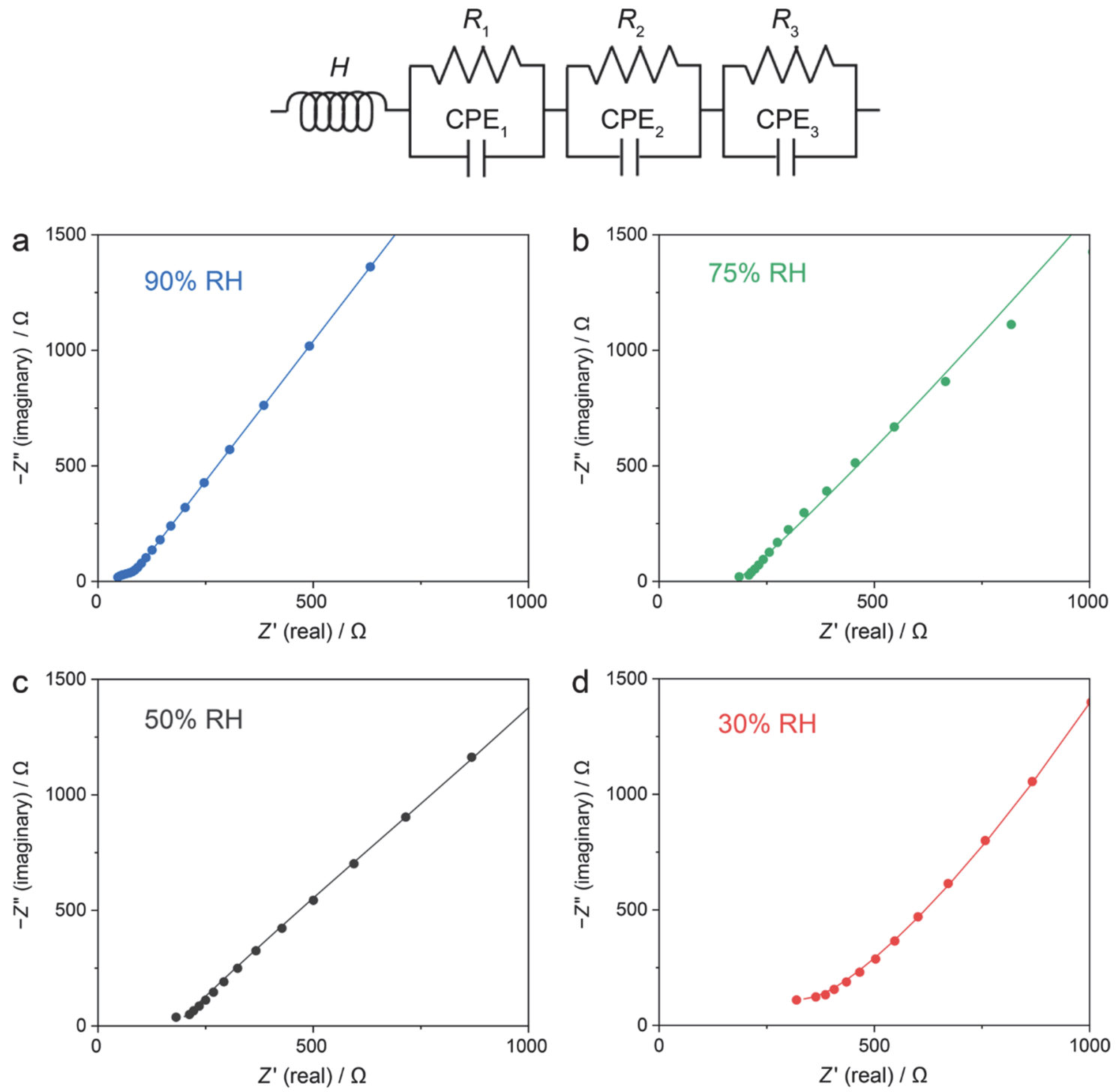

Figure S11. Nyquist plots (solid circles) of the impedance spectra along with the fitting with an electrical equivalent circuit (see above and experimental section, solid lines) of a pelleted sample of II at $368 \mathrm{~K}$ and RH of (a) $90 \%$, (b) $75 \%$ (c) $50 \%$, and (d) $30 \%$.

Bulk conductivities were estimated by a semicircle fitting of Nyquist plots. An electrical equivalent circuit (see above), which consists of a series of inductance $(H$, representing the effect of the external circuit) and three blocks of a resistance $(R)$ and constant phase element (used for imperfect capacitors) in parallel, standing for bulk, grain boundary, and electrode interface, was used to fit the impedance spectra. 


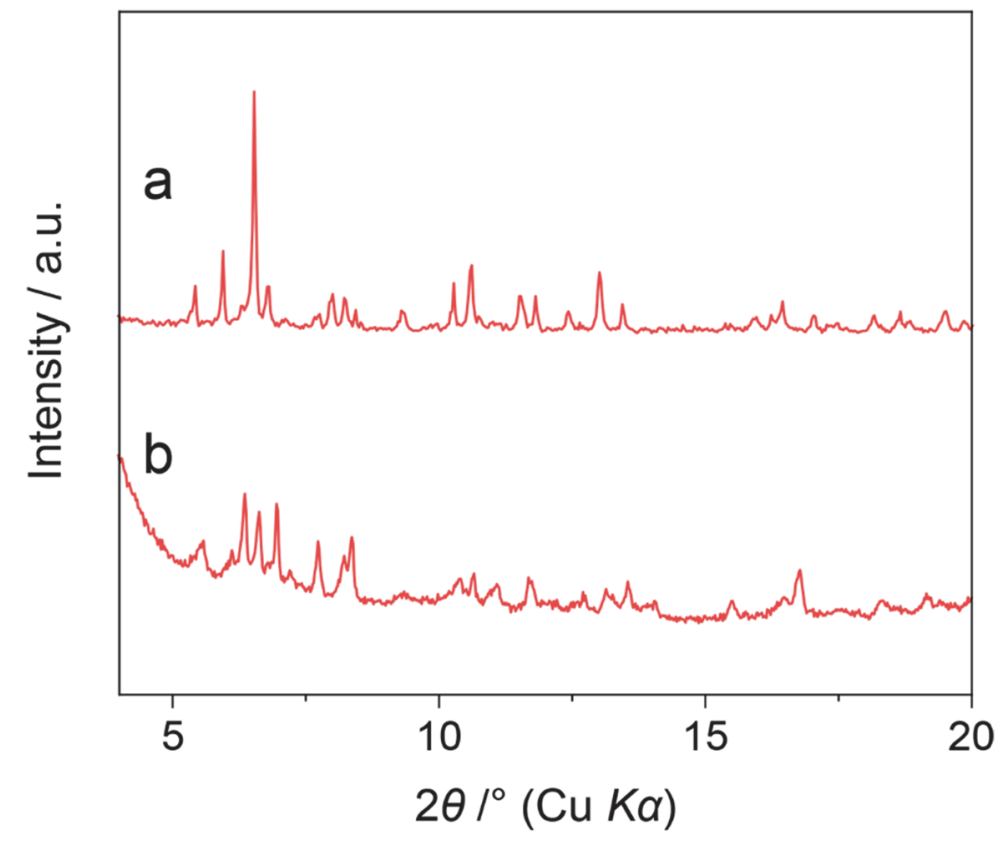

Figure S12. PXRD patterns of II (a) before and (b) after heating at $368 \mathrm{~K}$ and $90 \% \mathrm{RH}$.

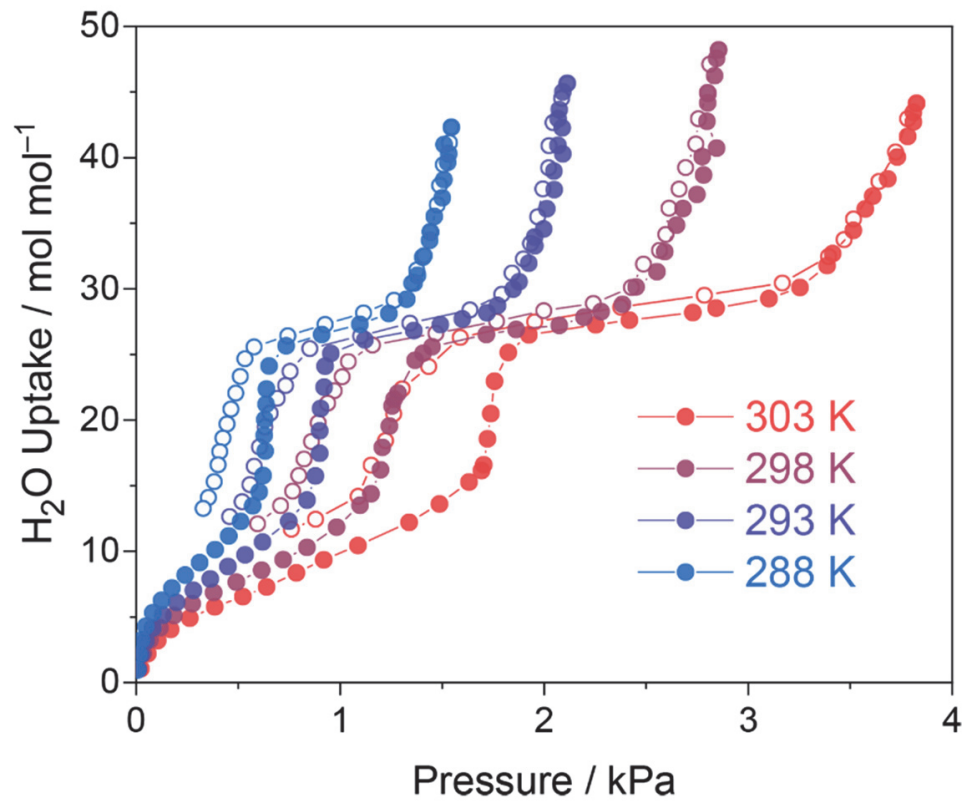

Figure S13. Water sorption-desorption isotherms of III. Solid and open circles show the sorption and desorption branch, respectively. 


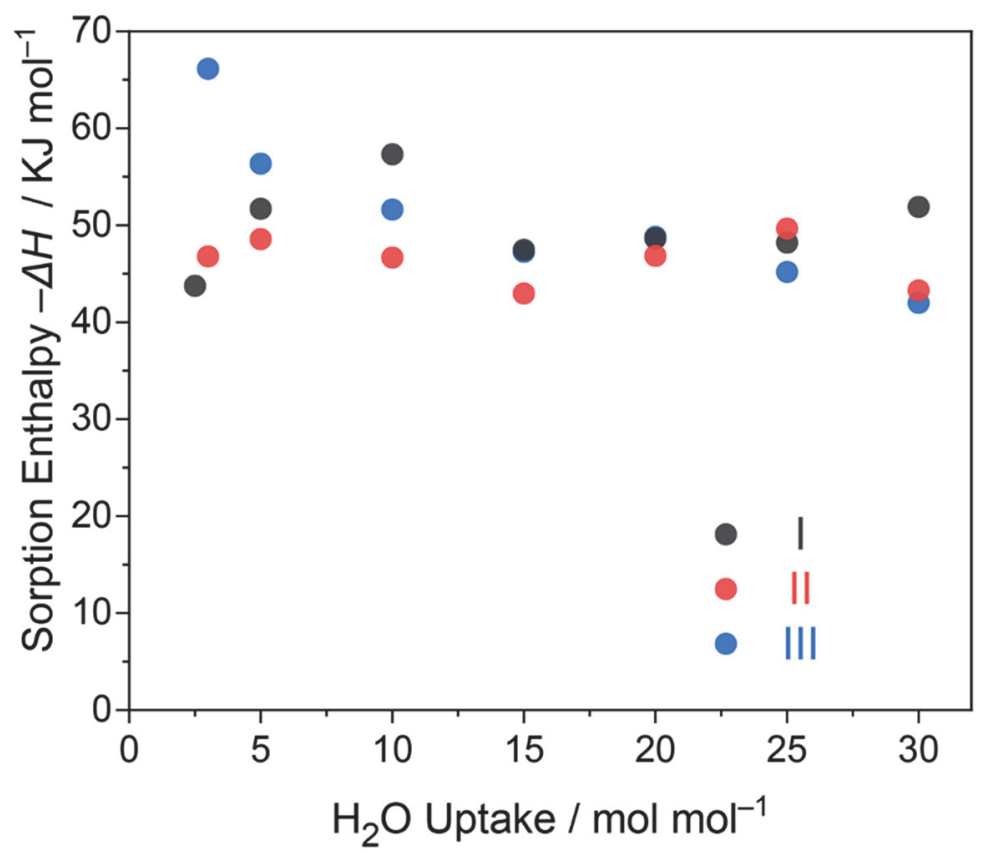

Figure S14. Changes in the water sorption enthalpy of I (black), II (red), and III (blue) with $\mathrm{H}_{2} \mathrm{O}$ uptake.

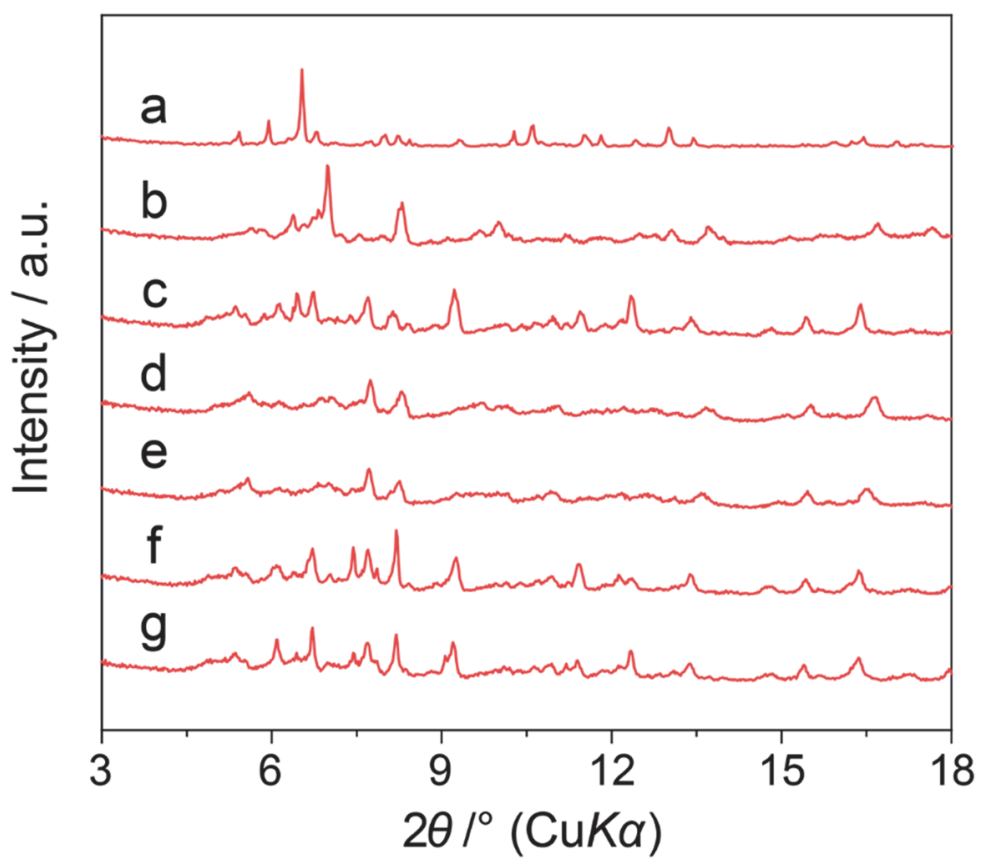

Figure S15. In situ PXRD patterns of II measured by the parallel beam method at 303 K. (a)(b) As synthesized, (c) dry $\mathrm{N}_{2}$, (d) RH 30\% (e) RH 50\%, (f) RH 75\%, and (g) RH ca. 100\%. Note that (a) is measured by the parafocusing method for comparison between the different methods. 


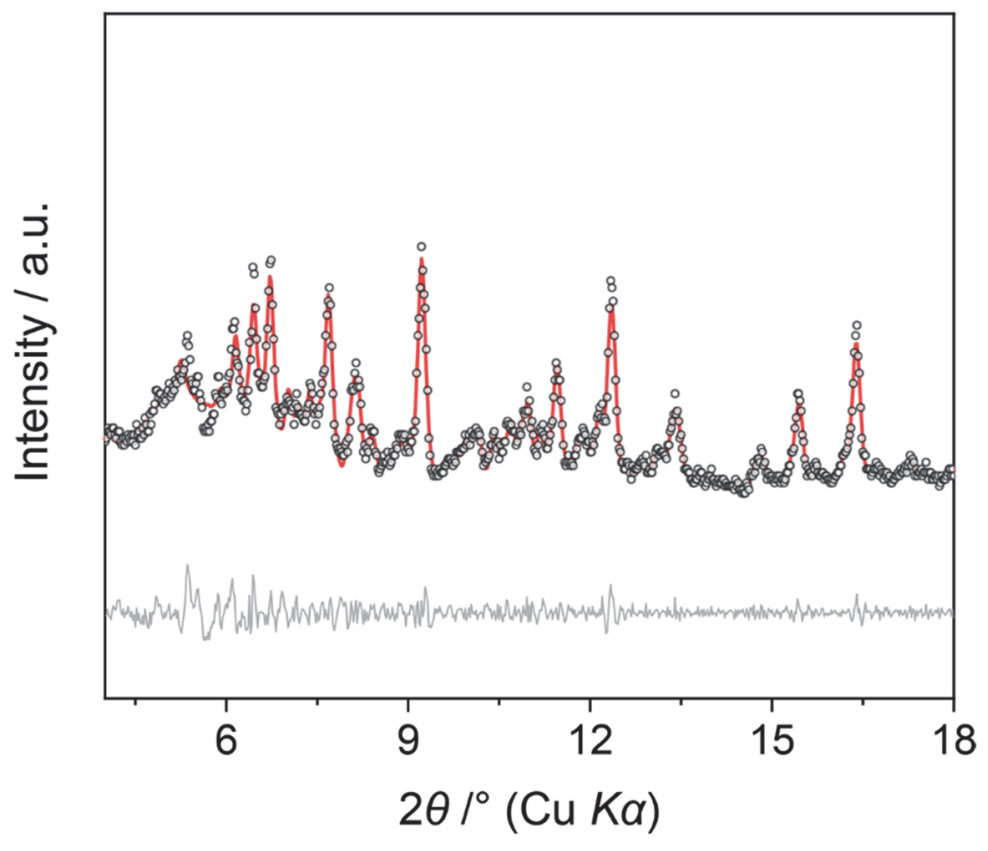

Figure S16. Experimental (open circles) and calculated (red solid line) PXRD patterns of II under $\mathrm{N}_{2}$ by the Pawley method. The bottom line shows the difference profile.

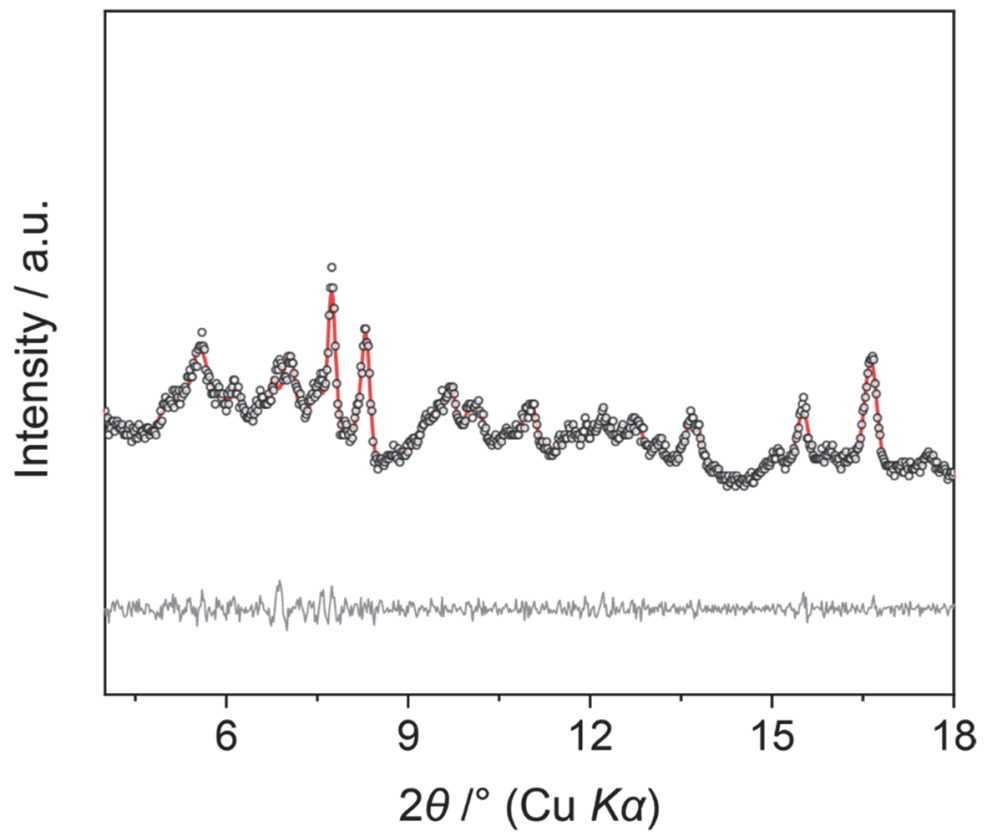

Figure S17. Experimental (open circles) and calculated (red solid line) PXRD patterns of II at RH of $30 \%$ by the Pawley method. The bottom line shows the difference profile. 


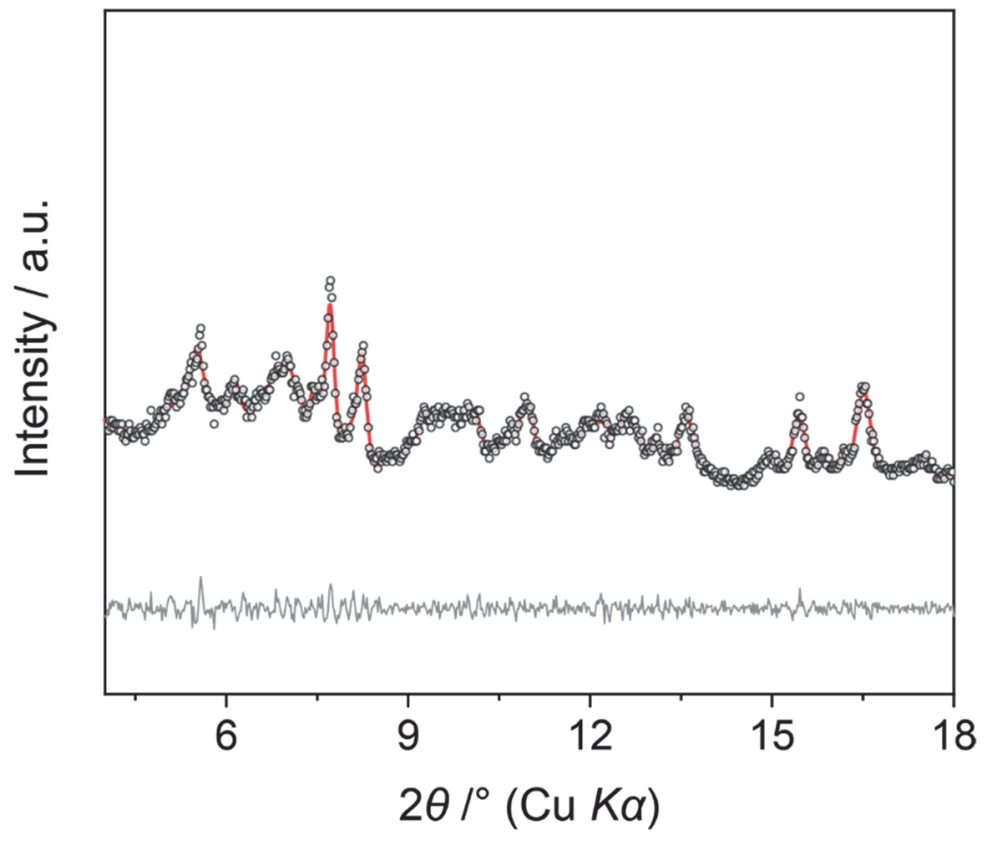

Figure S18. Experimental (open circles) and calculated (red solid line) PXRD patterns of II at RH of $50 \%$ by the Pawley method. The bottom line shows the difference profile.

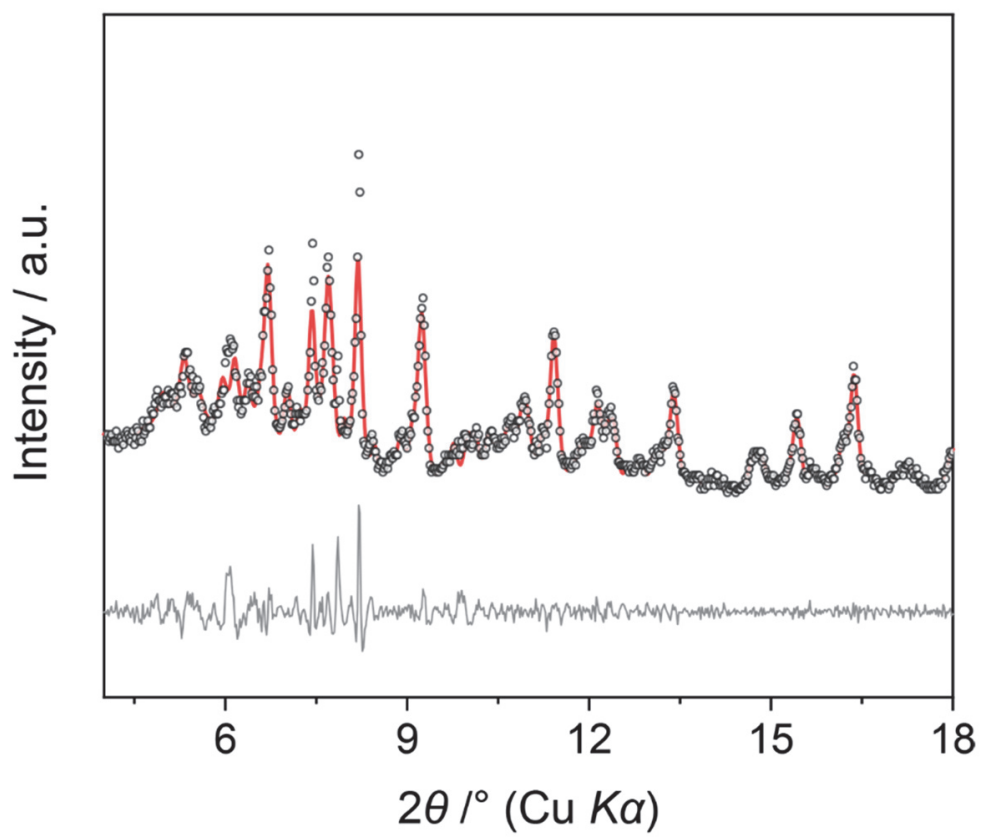

Figure S19. Experimental (open circles) and calculated (red solid line) PXRD patterns of II at RH of $75 \%$ by the Pawley method. The bottom line shows the difference profile. 


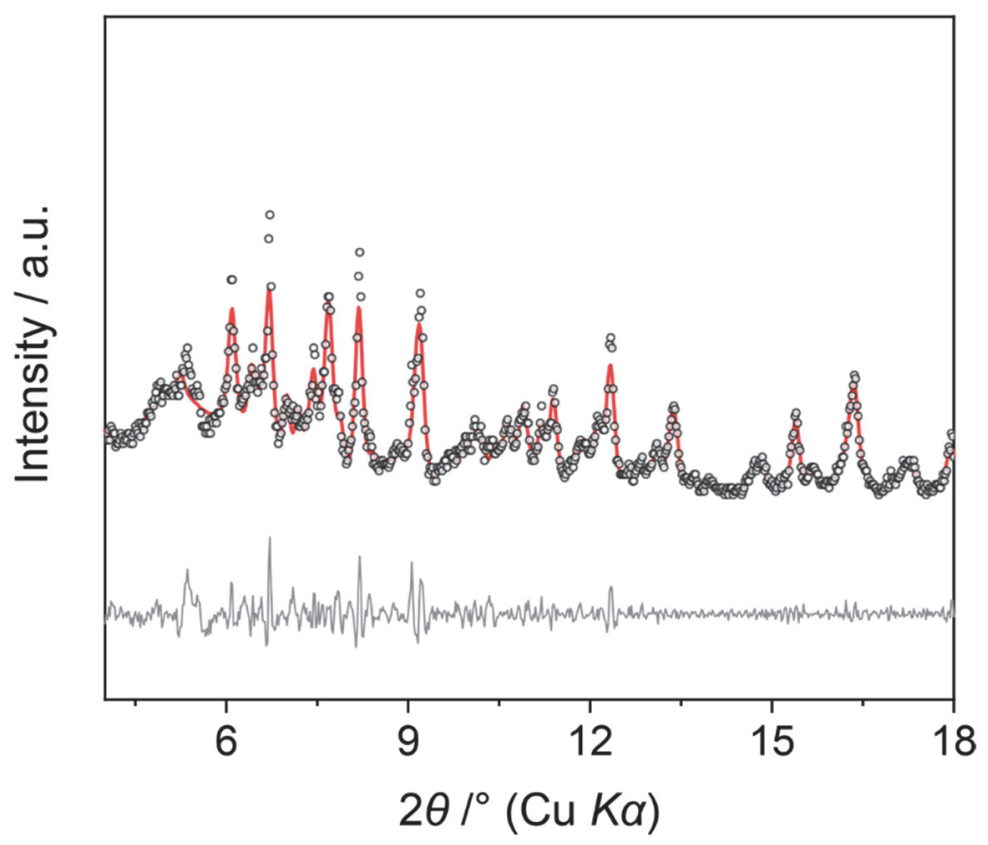

Figure S20. Experimental (open circles) and calculated (red solid line) PXRD patterns of II at RH of ca. $100 \%$ by the Pawley method. The bottom line shows the difference profile. 


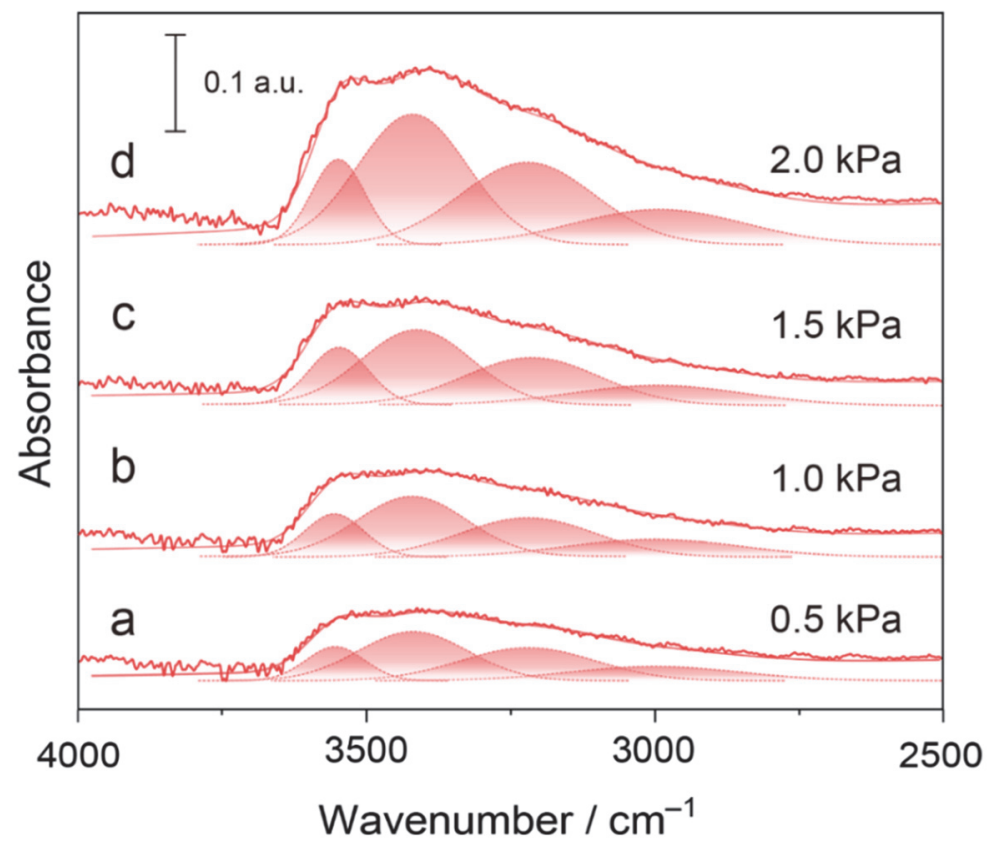

Figure S21. Background-subtracted IR spectra (293 K) of II under a water vapor pressure of (a) $0.5 \mathrm{kPa}$, (b) $1.0 \mathrm{kPa}$, (c) $1.5 \mathrm{kPa}$, and (d) $2.0 \mathrm{kPa}$ in the $\mathrm{OH}$ stretching region. The observed bands were well reproduced by the sum (solid lines) of four Gaussian peaks (broken lines). The peak area ratio among the four peaks did not change with the water vapor pressure.

The peak area ratio of the four bands (i:ii:iii:iv) for I, II, and III was similar to each other, i.e., 13:40:31:16, 12:39:34:15, and 12:40:28:19, respectively. According to the water sorption isotherms in Figures 5 and S13, all compounds sorb about $30 \mathrm{~mol} \mathrm{~mol}^{-1}$ of water molecules around this water vapor pressure $\left(P / P_{0}=0.85\right)$. Therefore, half of the water molecules (ca. $15 \mathrm{~mol} \mathrm{~mol}^{-1}$ ) in II should be interacting with $\mathrm{K}^{+}$or $\mathrm{Eu}^{3+}$. Considering that $\mathrm{Eu}^{3+}$ is coordinated by eight water molecules (see XAFS, Figure 3 and Table 1), the remaining seven water molecules are probably interacting with $\mathrm{K}^{+}$, which is in accord with the number and coordination environment of $\mathrm{K}^{+}$. 


\section{References}

1. Yang, Y.; He, X.; Zhang, P.; Andaloussi, Y. H.; Zhang, H.; Jiang, Z.; Chen, Y.; Ma, S.; Cheng, P.; Zhang, Z. Combined Intrinsic and Extrinsic Proton Conduction in Robust Covalent Organic Frameworks for Hydrogen Fuel Cell Applications. Angew. Chem. Int. Ed. 2020, 59, 3678-3684.

2. Yang, P.; Alsufyani, M.; Emwas, A. H.; Chen, C.; Khashab, N. M. Lewis Acid Guests in a $\left\{\mathrm{P}_{8} \mathrm{~W}_{48}\right\}$ Archetypal Polyoxotungstate Host: Enhanced Proton Conductivity via Metal-Oxo Cluster within Cluster Assemblies. Angew. Chem. Int. Ed. 2018, 57, 13046-13051.

3. Sarango-Ramírez, M. K.; Lim, D. W.; Kolokolov, D. I.; Khudozhitkov, A. E.; Stepanov, A. G.; Kitagawa, H. Superprotonic Conductivity in Metal-Organic Framework via Solvent-Free Coordinative Urea Insertion. J. Am. Chem. Soc. 2020, 142, 6861-6865.

4. Lai, X.; Liu, Y.; Yang, G.; Liu, S.; Shi, Z.; Lu, Y.; Luo, F.; Liu, S. Controllable ProtonConducting Pathways: Via Situating Polyoxometalates in Targeting Pores of a MetalOrganic Framework. J. Mater. Chem. A 2017, 5, 9611-9617.

5. Li, Z.; Li, X. X.; Yang, T.; Cai, Z. W.; Zheng, S. T. Four-Shell Polyoxometalates Featuring High-Nuclearity Ln 26 Clusters: Structural Transformations of Nanoclusters into Frameworks Triggered by Transition-Metal Ions. Angew. Chem. Int. Ed. 2017, 56, 2664 2669.

6. Peng, Q.; Li, S.; Wang, R.; Liu, S.; Xie, L.; Zhai, J.; Zhang, J.; Zhao, Q.; Chen, X. Lanthanide Derivatives of Ta/W Mixed-Addendum POMs as Proton-Conducting Materials. Dalton Trans. 2017, 46, 4157-4160.

7. Zhang, F. M.; Dong, L. Z.; Qin, J. S.; Guan, W.; Liu, J.; Li, S. L.; Lu, M.; Lan, Y. Q.; Su, Z. M.; Zhou, H. C. Effect of Imidazole Arrangements on Proton-Conductivity in Metal-Organic Frameworks. J. Am. Chem. Soc. 2017, 139, 6183-6189.

8. Iwano, T.; Miyazawa, S.; Osuga, R.; Kondo, J. N.; Honjo, K.; Kitao, T.; Uemura, T.; Uchida, S. Confinement of Poly(Allylamine) in Preyssler-Type Polyoxometalate and Potassium Ion Framework for Enhanced Proton Conductivity. Commun. Chem. 2019, 2, 1-8.

9. Wong, N. E.; Ramaswamy, P.; Lee, A. S.; Gelfand, B. S.; Bladek, K. J.; Taylor, J. M.; Spasyuk, D. M.; Shimizu, G. K. H. Tuning Intrinsic and Extrinsic Proton Conduction in Metal-Organic Frameworks by the Lanthanide Contraction. J. Am. Chem. Soc. 2017, 139, 14676-14683.

10. Ma, H.; Liu, B.; Li, B.; Zhang, L.; Li, Y. G.; Tan, H. Q.; Zang, H. Y.; Zhu, G. Cationic Covalent Organic Frameworks: A Simple Platform of Anionic Exchange for Porosity Tuning and Proton Conduction. J. Am. Chem. Soc. 2016, 138, 5897-5903.

11. Liu, J. C.; Han, Q.; Chen, L. J.; Zhao, J. W.; Streb, C.; Song, Y. F. Aggregation of Giant Cerium-Bismuth Tungstate Clusters into a 3D Porous Framework with High Proton Conductivity. Angew. Chem. Int. Ed. 2018, 57, 8416-8420. 
12. He, L.; Nath, J. K.; Chen, E. X.; Lai, H. D.; Huang, S. L.; Lin, Q. Dual-Cubic-Cage Based Lanthanide Sulfate-Carboxylpyrazolate Frameworks with High Hydrolytic Stability and Remarkable Proton Conduction. Chem. Commun. 2019, 55, 2497-2500.

13. Iwano, T.; Miyazawa, S.; Uchida, S., Effect of Molecular Weights of Confined Single-Chain Poly(allylamine) toward Proton Conduction in Inorganic Frameworks based on PreysslerType Polyoxometalate. Inorg. Chim. Acta 2020, 499, 119204.

14. Hayashi, A.; Wihadi, M. N. K.; Ota, H.; Lopez, X.; Ichihashi, K.; Nishihara, S.; Inoue, K.; Tsunoji, N.; Sano, T.; Sadakane, M., Preparation of Preyssler-Type Phosphotungstate with One Central Potassium Cation and Potassium Cation Migration into the Preyssler Molecule to form Di-Potassium-Encapsulated Derivative. ACS Omega 2018, 3, 2363-2373.

15. Connors, K. A., Binding Constants: The Measurement of Molecular Complex Stability, Wiley-Interscience: New York, 1987. 